\title{
Satisfiability of ECTL* with tree constraints ${ }^{\star}$
}

\author{
Claudia Carapelle ${ }^{1}$, Shiguang Feng ${ }^{1}$, Alexander Kartzow ${ }^{2}$ and Markus Lohrey ${ }^{2}$ \\ 1 Institut für Informatik, Universität Leipzig, Germany \\ 2 Department für Elektrotechnik und Informatik, Universität Siegen, Germany
}

\begin{abstract}
Recently, we have shown that satisfiability for ECTL* with constraints over $\mathbb{Z}$ is decidable using a new technique. This approach reduces the satisfiability problem of ECTL* with constraints over some structure $\mathcal{A}$ (or class of structures) to the problem whether $\mathcal{A}$ has a certain model theoretic property that we called EHD (for "existence of homomorphisms is decidable"). Here we apply this approach to concrete domains that are tree-like and obtain several results. We show that satisfiability of ECTL* with constraints is decidable over (i) semi-linear orders (i.e., tree-like structures where branches form arbitrary linear orders), (ii) ordinal trees (semi-linear orders where the branches form ordinals), and (iii) infinitely branching trees of height $h$ for each fixed $h \in \mathbb{N}$. We prove that all these classes of structures have the property EHD. In contrast, we introduce Ehrenfeucht-Fraïssé-games for WMSO + B (weak MSO with the bounding quantifier) and use them to show that the infinite (order) tree does not have property EHD. As a consequence, a different approach has to be taken in order to settle the question whether satisfiability of $\mathrm{ECTL}^{*}$ (or even LTL) with constraints over the infinite (order) tree is decidable.
\end{abstract}

\section{Introduction}

Temporal logics like LTL, CTL or CTL* are nowadays standard languages for specifying system properties in verification. These logics are interpreted over node labeled graphs (Kripke structures), where the node labels (also called atomic propositions) represent abstract properties of a system. Clearly, such an abstracted system state does in general not contain all the information of the original system state. Consider for instance a program that manipulates two integer variables $x$ and $y$. A useful abstraction might be to introduce atomic propositions $v_{-2^{32}}, \ldots, v_{2^{32}}$ for $v \in\{x, y\}$, where the meaning of $v_{k}$ for $-2^{32}<k<2^{32}$ is that the variable $v \in\{x, y\}$ currently holds the value $k$, and $v_{-2^{32}}$ (resp., $v_{2^{32}}$ ) means that the current value of $v$ is at most $-2^{32}$ (resp., at least $2^{32}$ ). It is evident that such an abstraction might lead to incorrect results in model-checking.

To overcome this problem, extensions of temporal logics with constraints have been studied. In this setting, a model of a formula is not only a Kripke structure but a Kripke structure where every node is assigned several values from some

\footnotetext{
* This work is supported by the DFG Research Training Group 1763 (QuantLA) and the DFG research project GELO.
} 
fixed structure $\mathcal{C}$ (called a concrete domain). The logic is then enriched in such a way that it has access to the relations of the concrete domain. For instance, if $\mathcal{C}=(\mathbb{Z},=)$ then every node of the Kripke structure gets assigned several integers and the logic can compare the values assigned to neighboring nodes for equality.

In a recent paper [6] we introduced a new method (called EHD-method in the following) which shows decidability of the satisfiability problem for $\mathrm{CTL}^{*}$ extended by local constraints over the integers. The latter logic was first introduced in 8. In [7] we extended the EHD-method to extended computation tree logic $\left(\mathrm{ECTL}^{*}\right.$ ) with constraints over the integers (a powerful temporal logic that properly extends $\mathrm{CTL}^{*}$ ) and proved that satisfiability is still decidable. This result greatly improves the partial results on fragments of $\mathrm{CTL}^{*}$ obtained by Bozzelli, Gascon and Pinchinat 4/5/11.

The idea of the EHD-method is as follows. Let $\mathcal{C}$ be any concrete domain over a relational signature $\sigma$. Satisfiability of ECTL ${ }^{*}$ with constraints over $\mathcal{C}$ is decidable if $\mathcal{C}$ has the following two properties:

1. The structure $\mathcal{C}$ is negation-closed, i.e., the complement of any relation $R \in \sigma$ is definable in positive existential first-order logic.

2. There is a logic $\mathcal{L}$ with certain properties (listed below) and an $\mathcal{L}$-sentence $\varphi$ characterizing homomorphism to $\mathcal{C}$ in the sense that for any countable $\sigma$-structure $\mathcal{A}$ there is a homomorphism from $\mathcal{A}$ to $\mathcal{C}$ if and only if $\mathcal{A} \models \varphi$.

For the candidate logics $\mathcal{L}$ we need the following properties:

1. Satisfiability of a given $\mathcal{L}$-sentence over the class of infinite node-labeled trees is decidable.

2. $\mathcal{L}$ is closed under boolean combinations with monadic second-order formulas (MSO).

3. $\mathcal{L}$ is compatible with one dimensional first-order interpretations and with the $k$-copy operation, see 7 .

The most powerful logic with these properties that we are aware of is the set of all Boolean combinations of MSO-formulas and WMSO+B-formulas (briefly $\mathrm{Bool}(\mathrm{MSO}, \mathrm{WMSO}+\mathrm{B})$ ), where $\mathrm{WMSO}+\mathrm{B}$ is weak monadic second-order logic with the bounding quantifier. The bounding quantifier allows to express that there is a bound on the size of finite sets satisfying a certain property. Satisfiability of WMSO + B over infinite node-labeled trees was shown to be decidable in [1] (in contrast, decidability of full monadic second-order logic with the bounding quantifier over infinite node-labeled trees cannot be proved in ZFC [3] ). In [6] we proved that the existence of a homomorphism into $(\mathbb{Z},<,=)$ can be expressed in Bool(MSO, WMSO+B) (or even in $\mathrm{WMSO}+\mathrm{B}$ ).

These results gave rise to the hope that the EHD-method could also be fruitfully applied to other concrete domains. An interesting candidate in this setting is the full infinitely branching infinite (order) tree $\mathcal{T}_{\infty}=\left(\mathbb{N}^{*},<, \perp,=\right)$, where $<$ denotes the prefix order on $\mathbb{N}^{*}$ and $\perp$ denotes the incomparability relation with respect to $<$ (this structure is negation-closed, which is the reason for adding the incomparability relation $\perp$ ). Unfortunately, this hope is destroyed by one of the main results of this work: 
Theorem 1. There is no Bool(MSO, WMSO $+\mathrm{B})$-sentence $\psi$ such that for every countable structure $\mathcal{A}$ (over the signature $\{<, \perp,=\}$ ) we have: $\mathcal{A} \models \psi$ if and only if there is a homomorphism from $\mathcal{A}$ to $\mathcal{T}_{\infty}$.

This result is shown using a suitable Ehrenfeucht-Fraïssé-game.

Theorem 1 shows that the EHD-method cannot be applied to the concrete domain $\mathcal{T}_{\infty}$. Of course, this does not imply that satisfiability for ECTL ${ }^{*}$ with constraints over $\mathcal{T}_{\infty}$ is undecidable, which remains an open problem (even for LTL instead of ECTL*). In fact, we still conjecture that satisfiability for ECTL* with constraints over $\mathcal{T}_{\infty}$ is decidable and we support this conjecture by fruitfully applying the EHD-method to other tree-like structures, such as semi-linear orders, ordinal trees, and infinitely branching trees of a fixed height. Semi-linear orders are partial orders that are tree-like in the sense that for every element $x$ the set of all smaller elements form a linear suborder. If this linear suborder is an ordinal (for every $x$ ) then one has an ordinal tree. Ordinal trees are widely studied in descriptive set theory and recursion theory. Note that a tree is a connected semi-linear order where for every element the set of all smaller elements is a finite linear order.

In the integer-setting from [67, we investigated satisfiability for ECTL*formulas with constraints over one fixed structure (integers with additional relations). For semi-linear orders and ordinal trees it is more natural to consider satisfiability with respect to a class of concrete domains $\Gamma$ (over a fixed signature $\sigma$ ): The question becomes, whether for a given constraint ECTL* formula $\varphi$ there is a concrete domain $\mathcal{C} \in \Gamma$ such that $\varphi$ is satisfiable by some model with concrete values from $\mathcal{C}: 3$ If a class $\Gamma$ has a universal structure $\mathcal{U}$, then satisfiability with respect to the class $\Gamma$ is equivalent to satisfiability with respect to $\mathcal{U}$ because one easily shows that a formula $\varphi$ has a model with some concrete domain from $\Gamma$ if and only if it has a model with concrete domain $\mathcal{U}$. A typical class with a universal model is the class of all countable linear orders, for which $(\mathbb{Q},<)$ is universal. Similarly, for the class of all countable trees the infinitely branching infinite tree as well as the binary infinite tree are universal. In the appendix we construct a universal countable semi-linear order. Since this particular universal structure appears to be less natural than $(\mathbb{Q},<)$ or the infinite binary tree, we have decided to formulate our decidability result for the class of all semi-linear orders. Moreover, there is no universal structure for the class of countable ordinal trees (for a similar reason as the one showing that the class of countable ordinals does not contain a universal structure).

Application of the EHD-method to semi-linear orders and ordinal trees gives the following decidability results.

Theorem 2. Satisfiability of ECTL*-formulas with constraints over each of the following classes is decidable:

\footnotetext{
${ }^{3}$ If the class $\Gamma$ is closed under taking induced substructures (which is the case for our classes) then one can restrict $\Gamma$ to its countable members.

${ }^{4}$ A structure $\mathcal{U}$ is universal for a class $\Gamma$ if there is a homomorphic embedding of every structure from $\Gamma$ into $\mathcal{U}$ and $\mathcal{U}$ belongs to $\Gamma$.
} 
(1) the class of all semi-linear orders,

(2) the class of all ordinal trees, and

(3) for each $h \in \mathbb{N}$, the class of all order trees of height $h$.

Concerning computational complexity, let us remark that in 66] we did not present an upper bound on the complexity of our decision procedure. The reason for this is that the authors of [1] do not proof an upper bound for the complexity of satisfiability of WMSO+B over infinite trees, even in the case that the input formula has bounded quantifier depth (and it is not clear how to obtain such a bound from the proof of [1. Here, the situation is slightly different. Our applications of the EHD-method for the proof Theorem 2 do not need the bounding quantifier, and classical WMSO (for semi-linear orders) and MSO (for ordinal trees and trees of bounded height) suffice. Moreover, the formulas that express the existence of a homomorphism have only small quantifier depth (at least for semi-linear orders and ordinal trees; for trees of bounded height, the quantifier depth depends on the height). This fact can be exploited and yields a triply exponential upper bound on the time complexity in (1) and (2) from Theorem 2 (this bound does not match the doubly exponential lower bound inherited from the satisfiability problem of ECTL* without constraints). We skipped the proof details, since we conjecture the exact complexity to be doubly exponential.

The paper is organized as follows: Section 2 introduced the necessary machinery concerning Kripke structures, tree-like partial orders and the logics MSO, WMSO + B and ECTL* with constraints. Moreover, we explain the EHDmethod (more details can be found in [7]). Using the EHD-method, Theorem 2 is proved in Sections 3 (for semi-linear orders), 4 (for ordinal trees) and 5 (for trees of bounded height $h$ ). Section $[6$ introduces an Ehrenfeucht-Fraïssé-game for $\mathrm{WMSO}+\mathrm{B}$ and uses this game to prove Theorem 1 .

\section{Preliminaries}

In this section we introduce basic notations concerning Kripke structures, various classes of tree-like structures, and the logics MSO, WMSO+B, and ECTL* with constraints.

\section{$2.1 \quad$ Structures}

Let $\mathrm{P}$ be a countable set of (atomic) propositions. A Kripke structure (over $\mathrm{P}$ ) is a triple $\mathcal{K}=(D, \rightarrow, \rho)$, where:

- $D$ is an arbitrary set of nodes (or states),

$-\rightarrow$ is a binary relation on $D$ such that for all $u \in D$ there exists $v \in D$ with $u \rightarrow v$, and

$-\rho: D \rightarrow 2^{\mathrm{P}}$ is a labeling function that assigns to every node a set of atomic propositions. 
A (finite relational) signature is a finite set $\sigma=\left\{r_{1}, \ldots, r_{n}\right\}$ of relation symbols. Every relation symbol $r \in \sigma$ has an associated arity $\operatorname{ar}(r) \geq 1$. A $\sigma$-structure is a pair $\mathcal{A}=(A, I)$, where $A$ is a non-empty set and $I$ maps every $r \in \sigma$ to an $\operatorname{ar}(r)$-ary relation over $A$. Quite often, we will identify the relation $I(r)$ with the relation symbol $r$, and we will specify a $\sigma$-structure as $\left(A, r_{1}, \ldots, r_{n}\right)$.

Given $\mathcal{A}=\left(A, r_{1}, \ldots, r_{n}\right)$ and given a subset $B$ of $A$, we define $r_{i \uparrow B}=$ $r_{i} \cap B^{\operatorname{ar}\left(r_{i}\right)}$ and $\mathcal{A}_{\uparrow B}=\left(B, r_{1 \uparrow B}, \ldots, r_{n \uparrow B}\right)$ (the restriction of $\mathcal{A}$ to the set $\left.B\right)$.

For a subsignature $\tau \subseteq \sigma$, a $\tau$-structure $\mathcal{B}=(B, J)$ and a $\sigma$-structure $\mathcal{A}=$ $(A, I)$, a homomorphism from $\mathcal{B}$ to $\mathcal{A}$ is a mapping $h: B \rightarrow A$ such that for all $r \in \tau$ and all tuples $\left(b_{1}, \ldots, b_{\operatorname{ar}(r)}\right) \in J(r)$ we have $\left(h\left(b_{1}\right), \ldots, h\left(b_{\operatorname{ar}(r)}\right)\right) \in I(r)$. We write $\mathcal{B} \preceq \mathcal{A}$ if there is a homomorphism from $\mathcal{B}$ to $\mathcal{A}$. Note that we do not require this homomorphism to be injective.

We now introduce constraint graphs. These are two-sorted structures where one part is a Kripke structure and the other part is some $\sigma$-structure called the concrete domain. To connect the concrete domain with the Kripke structure, we fix a set of unary function symbols $\mathcal{F}$. The interpretation of a function symbol from $\mathcal{F}$ is a mapping from the states of the Kripke structure to the universe of the concrete domain. Constraint graphs are the structures in which we evaluate constraint ECTL ${ }^{*}$-formulas.

Definition 3. An $\mathcal{A}$-constraint graph $\mathbb{C}$ is a tuple $\left(\mathcal{A}, \mathcal{K},\left(f^{\mathbb{C}}\right)_{f \in \mathcal{F}}\right)$ where:

- $\mathcal{A}=(A, I)$ is a $\sigma$-structure (the concrete domain),

$-\mathcal{K}=(D, \rightarrow, \rho)$ is a Kripke structure, and

- for each $f \in \mathcal{F}, f^{\mathbb{C}}: D \rightarrow A$ is the interpretation of the function symbol $f$ connecting elements of the Kripke structure with elements of the concrete domain.

Definition 4. An $\mathcal{A}$-constraint path $\mathbb{P}$ is an $\mathcal{A}$-constraint graph of the form $\mathbb{P}=\left(\mathcal{A}, \mathcal{P},\left(f^{\mathbb{P}}\right)_{f \in \mathcal{F}}\right)$, where $\mathcal{P}=(\mathbb{N}, \mathrm{S}, \rho)$ is a Kripke structure such that $\mathrm{S}$ is the successor relation on $\mathbb{N}$.

We use $\left(\mathcal{A}, \mathcal{K}, \mathcal{F}^{\mathbb{C}}\right)$ as an abbreviation for $\left(\mathcal{A}, \mathcal{K},\left(f^{\mathbb{C}}\right)_{f \in \mathcal{F}}\right)$. Moreover, we often drop the superscript $\mathbb{C}$ and also write constraint graph instead of $\mathcal{A}$-constraint graph if no confusion arises.

\subsection{Tree-like Structures}

We now introduce trees in the sense of Wolk [16, which are also known as semilinear orders. They are partial orders $\mathcal{P}=(P,<)$ with the additional property that for all $p \in P$ the suborder induced by $\left\{p^{\prime} \in P \mid p^{\prime} \leq p\right\}$ forms a linear order. This property is equivalent to the one formulated by Wolk [16]: Given incomparable elements $p_{1}, p_{2} \in P$, there is no $q \in P$ such that $p_{1}<q$ and $p_{2}<q$, i.e., two incomparable elements cannot have a common descendant. Clearly all trees (in the usual sense) satisfy this property, but not vice-versa.

We call a semi-linear order $\mathcal{P}=(P,<)$ an ordinal forest (resp., forest) if for all $p \in P$ the suborder induced by $\left\{p^{\prime} \in P \mid p^{\prime} \leq p\right\}$ is an ordinal (resp., a finite 
linear order). A (ordinal) forest is a (ordinal) tree if it has a unique minimal element. A forest $\mathcal{F}$ of height $h$ (for $h \in \mathbb{N}$ ) is a forest that contains a linear suborder with $h+1$ many elements but no linear suborder with $h+2$ elements. We say that an element $x \in P$ is at level $i$ if $|\{y \in P \mid y<x\}|=i$. Thus, every minimal element is at level 0 .

Given a partial order $(P,<)$, we denote by $\perp_{<}$the incomparability relation defined by $p \perp_{<} q$ iff neither $p \leq q$ nor $q \leq p$. Given a $\{<, \perp,=\}$-structure $\mathcal{P}=(P,<, \perp,=)$ such that $(P,<)$ is a semi-linear order (resp., ordinal tree, tree of height $h),=$ is the equality relation on $P$, and $\perp=\perp_{<}$, then we also say that $\mathcal{P}$ is a semi-linear order (resp. ordinal tree, tree of height $h$ ).

Let us mention that the class of all countable semi-linear orders contains a universal structure (see Appendix A). On the other hand, the class of all countable ordinal trees does not contain a universal structure, but there is a fixed uncountable ordinal tree such that all countable ordinal trees embed into this uncountable ordinal tree.

\subsection{Logics}

Monadic second-order logic (MSO) is the extension of first-order logic where also quantification over subsets of the underlying structure is allowed. Let us fix a countably infinite set $\mathrm{Var}_{1}$ of first-order variables that range over elements of a structure and a countably infinite set $\operatorname{Var}_{2}$ of second-order variables that range over subsets of a structure. MSO-formulas over the signature $\sigma$ are given by the following grammar, where $r \in \sigma, x, y, x_{1}, \ldots, x_{\operatorname{ar}(r)} \in \operatorname{Var}_{1}$, and $X \in \operatorname{Var}_{2}$ :

$$
\varphi::=r\left(x_{1}, \ldots, x_{\operatorname{ar}(r)}\right)|x=y| x \in X|\neg \varphi|(\varphi \wedge \varphi)|\exists x \varphi| \exists X \varphi .
$$

Weak monadic second-order logic (WMSO) has the same syntax as MSO but second-order variables only range over finite subsets of the underlying structure.

Finally, WMSO $+\mathrm{B}$ is the extension of WMSO by the bounding quantifier $\mathrm{B} X \varphi$ (see [2]) whose semantics is given by $\mathcal{A} \models \mathrm{B} X \varphi(X)$ (with $\mathcal{A}=(A, I)$ ) if and only if there is a bound $b \in \mathbb{N}$ such that $|B| \leq b$ for every finite subset $B \subseteq A$ with $\mathcal{A} \models \varphi(B)$. The quantifier rank of a WMSO+B-formula is the maximal number of nested quantifiers (existential, universal, and bounding quantifiers) in the formula. With Bool(MSO, WMSO +B) we denote the set of all boolean combinations of MSO-formulas and $\mathrm{WMSO}+\mathrm{B}$-formulas.

Extended computation tree logic $\left(\mathrm{ECTL}^{*}\right)$ is a branching time temporal logic first introduced in 1415 as an extension of $C T L^{*}$. As the latter, ECTL* is interpreted on Kripke structures, but while $\mathrm{CTL}^{*}$ allows to specify LTL properties of infinite paths of such models, ECTL* can describe regular (i.e., MSO-definable) properties of paths. In [7] we introduced an extension of ECTL*, called constraint ECTL*, which enriches ECTL* by local constraints in path formulas.

We now first recall the definition of constraint path MSO-formulas, which take the role of path formulas in constraint ECTL*. Constraint path MSO (over a signature $\tau$ ), denoted as $\mathrm{MSO}(\tau)$, is the usual MSO for (colored) infinite paths (also known as word structures) with a successor function $\mathrm{S}$ extended by atomic 
formulas that describe local constraints over the concrete domain. Thus, given a $\tau$-structure $\mathcal{A}, \operatorname{MSO}(\tau)$ can be evaluated over the class of $\mathcal{A}$-constraint paths.

In this paper we exclusively consider tree-like concrete domains over the fixed signature $\tau=\{<, \perp,=\}$. Therefore, we simplify the presentation and introduce constraint path MSO over $\tau$ only. For a more general presentation we refer the reader to [7. So fix a set $\mathrm{P}$ of atomic propositions and a set $\mathcal{F}$ of unary function symbols. Terms and formulas of $\mathrm{MSO}(\tau)$ are defined by the following grammar:

$$
\begin{aligned}
t & ::=\mathrm{S}(t) \mid x \\
\psi & ::=p(x)\left|t=t^{\prime}\right| t \in X|\neg \psi|\left(\psi \wedge \psi^{\prime}\right)|\exists x \psi| \exists X \psi \mid f_{1} \mathrm{~S}^{i}(x) \circ f_{2} \mathrm{~S}^{j}(x)
\end{aligned}
$$

where $\circ \in \tau, t$ and $t^{\prime}$ are $\mathrm{MSO}(\tau)$-terms, $\psi$ and $\psi^{\prime}$ are $\mathrm{MSO}(\tau)$-formulas, $p \in \mathrm{P}$, $x \in \operatorname{Var}_{1}, X \in \operatorname{Var}_{2}, i, j \in \mathbb{N}$ and $f_{1}, f_{2} \in \mathcal{F}$. We call formulas of the form $f_{1} S^{i}(x) \circ f_{2} S^{j}(x)$ for $\circ \in \tau$ atomic constraints. It is important to notice that in an atomic constraint only one first-order variable $x$ is used.

Remark 5. Setting $\sigma=\{\mathrm{S}\} \cup \mathrm{P}$, where $\mathrm{S}$ is a unary function symbol and all elements of $\mathrm{P}$ are considered to be unary predicates, $\mathrm{MSO}(\tau)$ is $\mathrm{MSO}$ over $\sigma$ extended by atomic constraints over $\tau$.

Let $\mathbb{P}=\left(\mathcal{A}, \mathcal{P},\left(f^{\mathbb{P}}\right)_{f \in \mathcal{F}}\right)$ be an $\mathcal{A}$-constraint path where $\mathcal{P}=(\mathbb{N}, \mathrm{S}, \rho)$, and let $\eta:\left(\operatorname{Var}_{1} \cup \operatorname{Var}_{2}\right) \rightarrow\left(\mathbb{N} \cup 2^{\mathbb{N}}\right)$ be a valuation function mapping first-order variables to elements and second-order variables to sets. The satisfaction relation $\models$ is defined by structural induction as follows:

$$
\begin{aligned}
& (\mathbb{P}, \eta) \models p\left(\mathrm{~S}^{i}(x)\right) \text { iff } p \in \rho(\eta(x)+i) . \\
& (\mathbb{P}, \eta) \models \mathrm{S}^{i}\left(x_{1}\right)=\mathrm{S}^{j}\left(x_{2}\right) \text { iff } \eta\left(x_{1}\right)+i=\eta\left(x_{2}\right)+j . \\
& (\mathbb{P}, \eta) \models \mathrm{S}^{i}(x) \in X \text { iff } \eta(x)+i \in \eta(X) . \\
& (\mathbb{P}, \eta) \models \neg \psi \text { iff it is not the case that }(\mathbb{P}, \eta) \models \psi . \\
& (\mathbb{P}, \eta) \models\left(\psi_{1} \wedge \psi_{2}\right) \text { iff }(\mathbb{P}, \eta) \models \psi_{1} \text { and }(\mathbb{P}, \eta) \models \psi_{2} . \\
& (\mathbb{P}, \eta) \models \exists x \psi \text { iff there is an } n \in \mathbb{N} \text { such that }(\mathbb{P}, \eta[x \mapsto n]) \models \psi . \\
& (\mathbb{P}, \eta) \models \exists X \psi \text { iff there is an } E \subseteq \mathbb{N} \text { such that }(\mathbb{P}, \eta[X \mapsto E]) \models \psi . \\
& (\mathbb{P}, \eta) \models f_{1} \mathrm{~S}^{i}(x) \circ f_{2} \mathrm{~S}^{j}(x) \text { iff } \mathcal{A} \models f_{1}^{\mathbb{P}}(\rho(x)+i) \circ f_{2}^{\mathbb{P}}(\rho(x)+j) .
\end{aligned}
$$

For an $\operatorname{MSO}(\tau)$-formula $\psi$ the satisfaction relation only depends on the variables occurring freely in $\psi$. This motivates the following notation: If $\psi\left(X_{1}, \ldots, X_{m}\right)$ is an $\operatorname{MSO}(\tau)$-formula where $X_{1}, \ldots, X_{m} \in \operatorname{Var}_{2}$ are the only free variables, we write $\mathbb{P} \models \psi\left(A_{1}, \ldots, A_{m}\right)$ if and only if, for every valuation function $\eta$ such that $\eta\left(X_{i}\right)=A_{i}$, we have $(\mathbb{P}, \eta) \models \psi$.

Having defined $\mathrm{MSO}(\tau)$-formulas we are ready to define constraint ECTL* over the signature $\tau$ (denoted by $\operatorname{ECTL}^{*}(\tau)$ ):

$$
\varphi::=\mathrm{E} \psi(\underbrace{\varphi, \ldots, \varphi}_{m \text { times }})|(\varphi \wedge \varphi)| \neg \varphi
$$


where $\psi\left(X_{1}, \ldots, X_{m}\right)$ is an $\mathrm{MSO}(\tau)$-formula in which at most the second-order variables $X_{1}, \ldots, X_{m} \in \operatorname{Var}_{2}$ are allowed to occur freely.

$\mathrm{ECTL}^{*}(\tau)$-formulas are evaluated over nodes of $\mathcal{A}$-constraint graphs. Let $\mathbb{C}=$ $\left(\mathcal{A}, \mathcal{K},\left(f^{\mathbb{C}}\right)_{f \in \mathcal{F}}\right)$ be an $\mathcal{A}$-constraint graph, where $\mathcal{K}=(D, \rightarrow, \rho)$. We define an infinite path $\pi$ in $\mathcal{K}$ as a mapping $\pi: \mathbb{N} \rightarrow D$ such that $\pi(i) \rightarrow \pi(i+1)$ for all $i \geq 0$. For an infinite path $\pi$ in $\mathcal{K}$ we define the infinite constraint path $\mathbb{P}_{\pi}=\left(\mathcal{A},\left(\mathbb{N}, \mathrm{S}, \rho^{\prime}\right),\left(f^{\mathbb{P}_{\pi}}\right)_{f \in \mathcal{F}}\right)$, where $\rho^{\prime}(n)=\rho(\pi(n))$ and $f^{\mathbb{P}_{\pi}}(n)=f^{\mathbb{C}}(\pi(n))$. Note that we may have $\pi(i)=\pi(j)$ for $i \neq j$. Given $d \in D$ and an $\operatorname{ECTL}^{*}(\tau)$ formula $\varphi$, we define $(\mathbb{C}, d) \models \varphi$ inductively as follows:

- $(\mathbb{C}, d) \models \varphi_{1} \wedge \varphi_{2}$ iff $(\mathbb{C}, d) \models \varphi_{1}$ and $(\mathbb{C}, d) \models \varphi_{2}$.

- $(\mathbb{C}, d) \models \neg \varphi$ iff it is not the case that $(\mathbb{C}, d) \models \varphi$.

- $(\mathbb{C}, d) \models \mathrm{E} \psi\left(\varphi_{1}, \ldots, \varphi_{m}\right)$ iff there is an infinite path $\pi$ in $\mathcal{K}$ with $d=\pi(0)$ and $\mathbb{P}_{\pi} \models \psi\left(A_{1}, \ldots, A_{m}\right)$ where $A_{i}=\left\{j \mid j \geq 0,(\mathbb{C}, \pi(j)) \models \varphi_{i}\right\}$.

Note that for checking $(\mathbb{C}, d) \models \varphi$ we may ignore all propositions $p \in \mathrm{P}$ and all functions $f \in \mathcal{F}$ that do not occur in $\varphi$.

Given a class of $\tau$-structures $\Gamma$, SAT-ECTL $(\Gamma)$ denotes the following computational problem: Given a formula $\varphi \in \mathrm{ECTL}^{*}(\tau)$, is there a concrete domain $\mathcal{A} \in \Gamma$ and a constraint graph $\mathbb{C}=\left(\mathcal{A}, \mathcal{K},\left(f^{\mathbb{C}}\right)_{f \in \mathcal{F}}\right)$ such that $\mathbb{C} \models \varphi$ ? We also write $\operatorname{SAT}^{-\mathrm{ECTL}^{*}(\mathcal{A}) \text { instead of SAT-ECTL }}(\{\mathcal{A}\})$.

\subsection{Constraint ECTL* and Definable Homomorphisms}

Remember that we focus our interest in this paper on the satisfiability problem with respect to a class of structures over the signature $\tau=\{<, \perp,=\}$ where $=$ is always interpreted as equality and $\perp$ as the incomparability relation with respect to $<$. In $\left[7\right.$, we provided a connection between $\operatorname{SAT}^{-E C T L}{ }^{*}(\mathcal{A})$ for some $\tau$-structure $\mathcal{A}$ and the definability of homomorphisms to $\mathcal{A}$ in the logic Bool(MSO, WMSO $+\mathrm{B})$. To be more precise, we are interested in definability of homomorphisms to the $\{<, \perp\}$-reduct of $\mathcal{A}$. In order to facilitate the presentation of this connection, we fix a class $\Gamma$ of $\{<, \perp\}$-structures.

For every structure $\mathcal{A}=(A, I) \in \Gamma$ we denote by $\mathcal{A}^{=}$its expansion by equality, i.e., the $\tau$-structure $(A, J)$ where $J(<)=I(<), J(\perp)=I(\perp)$, and $J(=)=\{(a, a) \mid a \in A\}$. Similarly, we set $\Gamma^{=}=\left\{\mathcal{A}^{=} \mid \mathcal{A} \in \Gamma\right\}$.

We call $\Gamma^{=}$negation-closed if for every $r \in\{<, \perp,=\}$ there is a positive existential first-order formula $\varphi_{r}\left(x_{1}, \ldots, x_{\operatorname{ar}(r)}\right)$ (i.e., a formula that is built up from atomic formulas using $\wedge, \vee$, and $\exists$ ) such that for all $\mathcal{A}=(A, I) \in \Gamma$

$$
A^{\operatorname{ar}(r)} \backslash I(r)=\left\{\left(a_{1}, \ldots, a_{\operatorname{ar}(r)}\right) \mid \mathcal{A} \models \varphi_{r}\left(a_{1}, \ldots, a_{\operatorname{ar}(r)}\right)\right\} .
$$

In other words, the complement of every relation $I(r)$ must be definable by a positive existential first-order formula.

Example 6. For any class $\Delta$ of $\{<, \perp\}$-structures such that in every $\mathcal{A} \in \Delta$, (i) $<$ is interpreted as a strict partial order and (ii) $\perp$ is interpreted as the incomparability with respect to $<$ (i.e., $x \perp y$ iff neither $x \leq y$ nor $y \leq x$ ), $\Delta$ is 
negation-closed: For every $\mathcal{A} \in \Delta$ the following equalities hold in $\mathcal{A}^{=}$, where $A$ is the universe of $\mathcal{A}$ :

$$
\begin{aligned}
\left(A^{2} \backslash<\right) & =\{(x, y) \mid \mathcal{A} \models y<x \vee y=x \vee x \perp y\} \\
\left(A^{2} \backslash \perp\right) & =\{(x, y) \mid \mathcal{A} \models x<y \vee x=y \vee y<x\} \\
\left(A^{2} \backslash=\right) & =\{(x, y) \mid \mathcal{A} \models x<y \vee x \perp y \vee y<x\}
\end{aligned}
$$

In particular, the class of all semi-linear orders and all its subclasses are negationclosed. Note that for this it is crucial that we add the incomparability relation $\perp$.

Definition 7. We say that $\Gamma$ has the property EHD (existence of a homomorphism to a structure from $\Gamma$ is Bool(MSO, WMSO+B)-definable) if there is a Bool(MSO, WMSO+B)-sentence $\varphi$ such that for every countable $\{<, \perp\}$ structure $\mathcal{B}$

$$
\mathcal{B} \preceq \mathcal{A} \text { for some } \mathcal{A} \in \Gamma \quad \Longleftrightarrow \mathcal{B} \models \varphi \text {. }
$$

Now the following theorem connects $\operatorname{SAT}-\operatorname{ECTL}^{*}\left(\Gamma^{=}\right)$with EHD for the class $\Gamma$.

Theorem 8 ([7]). Let $\Gamma$ be a class of structures over $\{<, \perp\}$. If $\Gamma^{=}$is negationclosed and $\Gamma$ has EHD, then the problem SAT-ECTL $\left(\Gamma^{=}\right)$is decidable.

In the next sections, we show that the following classes of tree-like structures have EHD:

1. the class of all semi-linear orders,

2. the class of all ordinal trees, and

3. for each $h \in \mathbb{N}$ the class of all trees of height $h$.

Thus, Theorem 8 shows that for these classes, the satisfiability problems for $\mathrm{ECTL}^{*}$ with constraints are decidable, which proves our main Theorem 2 .

\section{Constraint ECTL* over Semi-Linear Orders}

Let $\Gamma$ denote the class of all semi-linear orders (over $\{<, \perp\}$ ). The aim of this section is to prove that $\Gamma$ has EHD. For this purpose, we characterize all those structures that admit a homomorphism to some element of $\Gamma$. The resulting criterion can be easily translated into WMSO. Hence, we do not need the bounding quantifier from WMSO + B here (the same will be true in the following Sections 4 and 5).

It turns out that, in the case of semi-linear orders (and also ordinal forests) the existence of such a homomorphism is in fact equivalent to the existence of a compatible expansion. We say that a graph $5(A,<, \perp)$ can be extended to a semilinear order (an ordinal forest) if there is a partial order $\triangleleft \operatorname{such}$ that $\left(A, \triangleleft, \perp_{\triangleleft}\right)$

\footnotetext{
${ }^{5}$ We call $(A,<, \perp)$ a graph to emphasize that here the binary relation symbols $<$ and $\perp$ can have arbitrary interpretations and they need not be a partial order and its incomparability relation. We can instead see them as two different kinds of edges in an arbitrary graph.
} 
is a semi-linear order (a ordinal forest) compatible with $(A,<, \perp)$, i.e.,

$$
x<y \Rightarrow x \triangleleft y \text { and } x \perp y \Rightarrow x \perp \triangleleft y .
$$

Lemma 9. The following are equivalent for every structure $\mathcal{A}=(A,<, \perp)$ :

1. $\mathcal{A}$ can be extended to a semi-linear order (to an ordinal forest, resp.).

2. $\mathcal{A} \preceq \mathcal{B}$ for some semi-linear order (ordinal tree, resp.) $\mathcal{B}$.

Proof. We start with the implication $(1 \Rightarrow 2)$. Assume that $\mathcal{A}$ can be extended to a compatible semi-linear order (ordinal forest, resp.) $\mathcal{A}^{\prime}=\left(A, \triangleleft, \perp_{\triangleleft}\right)$. Thanks to compatibility, the identity is a homomorphism from $\mathcal{A}$ to $\mathcal{A}^{\prime}$. In the case of an ordinal forest, one can add one common minimal element to obtain an ordinal tree.

Let us now prove $(2 \Rightarrow 1)$. Suppose $h$ is a homomorphism from $\mathcal{A}=(A,<, \perp)$ to some semi-linear order $\mathcal{B}=\left(B, \prec, \perp_{\prec}\right)$. We extend $\mathcal{A}$ to a compatible semilinear order $\left(A, \triangleleft, \perp_{\triangleleft}\right)$. Let us fix an arbitrary well-order $<_{\text {wo }}$ on the set $A$ (which exists by the axiom of choice). We define the binary relation $\triangleleft$ on $A$ as follows:

$$
x \triangleleft y \text { if and only if } h(x) \prec h(y) \text { or }\left(h(x)=h(y) \text { and } x<_{\text {wo }} y\right),
$$

As usual, we denote with $\perp_{\triangleleft}$ the incomparability relation for $\triangleleft$, i.e., $x \perp_{\triangleleft} y$ if and only if neither $x \triangleleft y$ nor $y \triangleleft x$ nor $x=y$ holds. We show that $\left(A, \triangleleft, \perp_{\triangleleft}\right)$ is a semi-linear order. In fact, irreflexivity and transitivity are easy consequences of the definition of $\triangleleft$ and of the fact that $\prec$ is a partial order. To show that $\triangleleft$ is semi-linear, assume that $x_{1} \triangleleft x$ and $x_{2} \triangleleft x$. By definition $h\left(x_{1}\right) \prec h(x)$ or $h\left(x_{1}\right)=h(x)$ and $h\left(x_{2}\right) \prec h(x)$ or $h\left(x_{2}\right)=h(x)$. By semi-linearity of $\mathcal{B}$, we deduce that $h\left(x_{1}\right)$ and $h\left(x_{2}\right)$ are comparable and, by definition of $\triangleleft$, so are $x_{1}$ and $x_{2}$. It remains to show that $\left(A, \triangleleft, \perp_{\triangleleft}\right)$ is compatible with $\mathcal{A}$. Let $x<y$. Then, by the fact that $h$ is a homomorphism, $h(x) \prec h(y)$ which guarantees that $x \triangleleft y$. If $x \perp y$, then $h(x) \perp_{\prec} h(y)$. Since $\mathcal{B}$ is a semi-linear order, this implies that neither $h(x) \prec h(y)$ nor $h(y) \prec h(x)$ nor $h(x)=h(y)$ holds. As a consequence none of $x \triangleleft y, y \triangleleft x$ and $x=y$ holds. Therefore we have $x \perp_{\triangleleft} y$.

The case in which $\mathcal{B}$ is an ordinal tree is dealt with similarly. It is enough to notice that $\triangleleft$ does not contain any infinite decreasing chains, since $\prec$ is wellfounded and $<_{\text {wo }}$ is a well-order.

Inspired by Wolk's work on comparability graphs 1617 we use Rado's selection lemma 13 in order to obtain the compactness result that a graph can be extended to a semi-linear order iff every finite subgraph is. Recall that a choice function for family of sets $X=\left\{X_{i} \mid i \in I\right\}$ is a function $f$ with domain $I$ such that $f(i) \in X_{i}$ for all $i \in I$, i.e., it chooses one element from each set $X_{i}$.

Lemma 10 (Rado's selection lemma, cf. [12,13]). Let I be an arbitrary index set and let $X=\left\{X_{i} \mid i \in I\right\}$ be a family of finite sets. For each finite subset $A$ of $I$, let $f_{A}$ be a choice function for the family $\left\{X_{i} \mid i \in A\right\}$. Then there is a choice function $f$ for $X$ such that, for all finite $A \subseteq I$, there is a finite set $B$ such that $A \subseteq B \subseteq I$ with $f(i)=f_{B}(i)$ for all $i \in A$. 
Lemma 11 (extension of Theorem 2 in [17]). A structure $\mathcal{A}=(A,<, \perp$ ) can be extended to a semi-linear order if and only if every finite substructure of $\mathcal{A}$ can be extended to a semi-linear order.

Proof. The direction $(\Rightarrow)$ is trivial. For the direction $(\Leftarrow)$, let

$$
I=\{\{x, y\} \subseteq A \mid x \neq y\}
$$

be the set of pairs of distinct elements of $A$. For all $i=\{x, y\} \in I$ we define $Z_{i}=\{(x, y),(y, x), \#\}$. We want to find a choice function for the family of sets $\left\{Z_{i} \mid i \in I\right\}$ which is in some sense compatible with the relations $\perp$ and $<$. In fact, choosing for each $i \in I$ one element of $Z_{i}$ corresponds intuitively to deciding whether the two elements $x$ and $y$ are comparable, and in which order, or if they are incomparable.

Each finite subset $J$ of $I$ defines a set $\bar{J}=\{x \in j \mid j \in J\}$ and a substructure $\mathcal{A}_{\uparrow \bar{J}}=\left(A_{\uparrow \bar{J}},<_{\uparrow \bar{J}}, \perp_{\uparrow \bar{J}}\right)$. Since $\mathcal{A}_{\uparrow \bar{J}}$ is finite, by hypothesis it can be extended to a semi-linear order. Hence, we can find a partial order $\triangleleft_{J}$ on $\bar{J}$ such that $\left(A_{\lceil\bar{J}}, \triangleleft_{J}, \perp_{\triangleleft_{J}}\right)$ is a semi-linear order compatible with $\mathcal{A}_{\uparrow \bar{J}}$ as in (2) on page 10 .

Let $f_{J}$ be the choice function for $\left\{Z_{j} \mid j \in J\right\}$ defined as follows:

$$
f_{J}(\{x, y\})=\left\{\begin{array}{cc}
(y, x) & \text { iff } y \triangleleft_{J} x, \\
(x, y) & \text { iff } x \triangleleft_{J} y, \\
\# & \text { otherwise. }
\end{array}\right.
$$

By Lemma 10 we can find a choice function $f$ for $\left\{Z_{i} \mid i \in I\right\}$ such that for all finite $J \subseteq I$ there is a finite set $K$ such that

$$
J \subseteq K \subseteq I \text { and } f(j)=f_{K}(j) \text { for all } j \in J .
$$

Define $x \triangleleft y$ iff $(x, y) \in f(I)$. We need to prove that $\left(A, \triangleleft, \perp_{\triangleleft}\right)$ is an extension of $\mathcal{A}$ to a semi-linear order. But all the properties that we need to check are local, and thanks to Rado's selection lemma, $\triangleleft$ always coincides, on every finite subset of $A$, with some $\triangleleft_{J}$, which is a semi-linear order compatible with $<$ and $\perp$.

Thanks to Lemma 11] given a $\{<, \perp\}$-structure $\mathcal{A}$, proving EHD only requires to look for a necessary and sufficient condition which guarantees that every finite substructure of $\mathcal{A}$ admits a homomorphism into a semi-linear order.

Definition 12. Let $\mathcal{A}=(A,<, \perp)$ be a graph. Given $A^{\prime} \subseteq A$, we say $A^{\prime}$ is connected (with respect to $<$ ) if and only if, for all $a, a^{\prime} \in A^{\prime}$, there are $a_{1}, \ldots, a_{n} \in A^{\prime}$ such that $a=a_{1}, a^{\prime}=a_{n}$ and $a_{i}<a_{i+1}$ or $a_{i+1}<a_{i}$ for all $1 \leq i \leq n-1$. A connected component of $\mathcal{A}$ is a maximal (with respect to inclusion) connected subset of $A$. Given a subset $A^{\prime} \subseteq A$ and $c \in A^{\prime}$, we say that $c$ is a central point of $A^{\prime}$ if and only if for every $a \in A^{\prime}$ neither $a \perp c$ nor $c \perp a$ nor $a<c$ holds.

In other words, a central point of a subset $A^{\prime} \subseteq A$ is a node of the structure $\mathcal{A}=(A,<, \perp)$ which has no incoming or outgoing $\perp$-edges, and no incoming $<$-edges within $A^{\prime}$. 

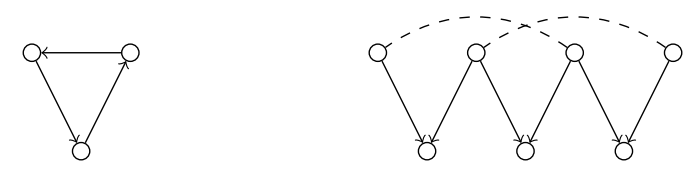

Fig. 1. A <-cycle of three elements and an "incomparable triple-u", where dashed lines are $\perp$-edges.

Example 13. A <-cycle (of any number of elements) does not have a central point, nor does an incomparable triple-u, see Figure 1. Both structures do not admit homomorphism into a semi-linear order. While this statement is obvious for the cycle, we leave the proof for the incomparable triple-u as an exercise.

Lemma 14. A finite structure $\mathcal{A}=(A,<, \perp)$ can be extended to a semi-linear order if and only if every non-empty connected $B \subseteq A$ has a central point.

Let us extract the main argument for the $(\Rightarrow)$-part of the proof for later reuse:

Lemma 15. Let $\left(A, \triangleleft, \perp_{\triangleleft}\right)$ be a semi-linear order extending $\mathcal{A}=(A,<, \perp)$. If a connected subset $B \subseteq A$ (with respect to $<$ ) contains a minimal element $m$ with respect to $\triangleleft$, then $m$ is central in $B$ (again with respect to $\mathcal{A}$ ).

Proof. Let $b \in B$. Since $B$ is connected, there are $b_{1}, \ldots, b_{n} \in B$ such that $b_{1}=m, b_{n}=b$ and $b_{i}<b_{i+1}$ or $b_{i+1}<b_{i}$ for all $1 \leq i \leq n-1$. As $\triangleleft$ is compatible with $<$, this implies that $b_{i} \triangleleft b_{i+1}$ or $b_{i+1} \triangleleft b_{i}$ for all $1 \leq i \leq n-1$. Given that $m$ is minimal, applying semi-linearity of $\triangleleft$, we obtain that $m=b_{i}$ or $m \triangleleft b_{i}$ for all $1 \leq i \leq n$. In particular, we have $m=b$ or $m \triangleleft b$. Since $\left(A, \triangleleft, \perp_{\triangleleft}\right)$ is a semi-linear order, compatible with $(A,<, \perp)$, we cannot have $b<m, m \perp b$ or $b \perp m$ (since this would imply $b \triangleleft m$ or $m \perp_{\triangleleft} b$ ). Hence, $m$ is central.

Proof (of Lemma 14). For the direction $(\Rightarrow$ ) let $B$ be any non-empty connected subset of $A$. Since $B$ is finite, there is a minimal element $m$. Using the previous lemma we conclude that $m$ is central in $B$.

We prove the direction $(\Leftarrow)$ by induction on $n=|A|$. Suppose $n=1$ and let $A=\{a\}$. The fact that $\{a\}$ has a central point implies that neither $a<a$ nor $a \perp a$ holds. Hence, $\mathcal{A}$ is a semi-linear order.

Suppose $n>1$ and assume the statement to be true for all $i<n$. If $\mathcal{A}$ is not connected with respect to $<$, then we apply the induction hypothesis to every connected component. The union of the resulting semi-linear orders extends $\mathcal{A}$. Now assume that $\mathcal{A}$ is connected and let $c$ be a central point of $A$. By the inductive hypothesis we can find $\triangleleft^{\prime}$ such that $\left(A \backslash\{c\}, \triangleleft^{\prime}, \perp \triangleleft^{\prime}\right)$ is a semi-linear order extending $\mathcal{A} \backslash\{c\}$. We define $\triangleleft:=\triangleleft^{\prime} \cup\{(c, x) \mid x \in A \backslash\{c\}\}$ (i.e., we add $c$ as a smallest element), which is obviously a partial order on $A$.

To prove that $\triangleleft$ is semi-linear, let $a_{1}, a_{2}, a \in A$ such that $a_{1} \triangleleft a$ and $a_{2} \triangleleft a$. If $a_{1}=c$ or $a_{2}=c$, then $a_{1}$ and $a_{2}$ are comparable by definition. Otherwise, we conclude that $a_{1}, a_{2}, a \in A \backslash\{c\}$. Hence, $a_{1} \triangleleft^{\prime} a$ and $a_{2} \triangleleft^{\prime} a$, and semi-linearity of $\triangleleft^{\prime}$ settles the claim. 
We finally show compatibility. Suppose that $a<b$. If $a=c$, then $a \triangleleft b$. The case $b=c$ cannot occur, because $c$ is central in $A$. The remaining possibility $a \neq c \neq b$ implies that $a \triangleleft^{\prime} b$ and hence $a \triangleleft b$ as desired. Finally, suppose that $a \perp b$. Then $a \neq c \neq b$, because $c$ is central. We conclude that $a \perp_{\triangleleft^{\prime}} b$ and also $a \perp_{\triangleleft} b$.

We are finally ready to state the main result of this section which (together with Theorem 8) completes the proof of the first part of Theorem 2

Proposition 16. The class of all semi-linear orders $\Gamma$ has $E H D$.

Proof. Take $\mathcal{A}=(A,<, \perp)$. Thanks to Lemmas 9, 11]and 14, it is enough to show that WMSO can express the condition that every finite and non-empty connected substructure of $\mathcal{A}$ has a central point. We define the following WMSO-formula $\operatorname{reach}(x, y, X)$ such that $\mathcal{A} \models \operatorname{reach}(a, b, B)$ if and only if $a$ and $b$ are in the same connected component of $\mathcal{A}_{\uparrow B}$ :

$$
x \in X \wedge \forall Y \subseteq X[(x \in Y \wedge \forall z \in Y \forall w \in X(\varphi(z, w) \rightarrow w \in Y)) \rightarrow y \in Y],
$$

where $\varphi(z, w):=z<w \vee w<z$. Then, we define the following WMSO-formulas:

$$
\begin{aligned}
\operatorname{connected}(X) & :=\forall x \in X \forall y \in X \operatorname{reach}(x, y, X), \\
\operatorname{central}(x, X) & :=x \in X \wedge \forall y \in X \neg(x \perp y \vee y \perp x \vee y<x), \text { and } \\
\psi & :=\forall X(\operatorname{connected}(X) \wedge X \neq \emptyset \rightarrow \exists x \operatorname{central}(x, X)) .
\end{aligned}
$$

It is straightforward to verify that $\mathcal{A} \models \psi$ if and only if every finite non-empty connected subset of $A$ has a central point.

\section{Constraint ECTL* over Ordinal Trees}

Let $\Omega$ denote the class of all ordinal trees (over the signature $\{<, \perp\}$ ). The aim of this section is to prove that $\Omega$ has EHD as well. We use again the notions of a connected subset and a central point as introduced in Definition 12. We will characterize those structures which admit a homomorphism into an ordinal tree. Here, in contrast with the case of semi-linear orders, the final condition will be that all connected sets (not just the finite ones) have a central point.

Lemma 17. Let $\mathcal{A}=(A,<, \perp)$ be a structure. There exists $\mathcal{O} \in \Omega$ such that $\mathcal{A} \preceq \mathcal{O}$ if and only if every non-empty and connected $B \subseteq A$ has a central point.

Proof. We start with the direction $(\Rightarrow)$. Due to Lemma 9 we can assume that there is a relation $\triangleleft$ that extends $(A,<, \perp)$ to an ordinal forest. Let $B \subseteq A$ be a non-empty connected set. Since $\left(A, \triangleleft, \perp_{\triangleleft}\right)$ is an ordinal forest, $B$ has a minimal element $c$ with respect to $\triangleleft$. By Lemma 15, $c$ is a central point of $B$.

For the direction $(\Leftarrow)$ we first define a partition of the domain of $\mathcal{A}$ into subsets $C_{\beta}$ for $\beta \sqsubset \chi$, where $\chi$ is an ordinal (whose cardinality is bounded by the cardinality of $A$ ). Here $\sqsubset$ denotes the natural order on ordinals. Assume that 
the pairwise disjoint subsets $C_{\beta}$ have been defined for all $\beta \sqsubset \alpha$ (which is true for $\alpha=0$ in the beginning). Then we define $C_{\alpha}$ as follows. Let $C_{\sqsubset \alpha}=\bigcup_{\beta \sqsubset \alpha} C_{\beta} \subseteq A$. If $A \backslash C_{\sqsubset \alpha}$ is not empty, then we define $\mathcal{C}_{\alpha}$ as the set of connected components of $A \backslash C_{\sqsubset \alpha}$. Let

$$
C_{\alpha}=\left\{c \in A \backslash C_{\sqsubset \alpha} \mid c \text { is a central point of some } B \in \mathcal{C}_{\alpha}\right\} .
$$

Clearly, $C_{\alpha}$ is not empty. Hence, there must exist a smallest ordinal $\chi$ such that $A=C_{\sqsubset \chi}$.

For every ordinal $\alpha \sqsubset \chi$ and each element $c \in C_{\alpha}$ we define the sequence of connected components $\operatorname{road}(c)=\left(B_{\beta}\right)_{(\beta \sqsubseteq \alpha)}$, where $B_{\beta} \in \mathcal{C}_{\beta}$ is the unique connected component with $c \in B_{\beta}$. This ordinal-indexed sequence keeps record of the road we took to reach $c$ by storing information about the connected components to which $c$ belongs at each stage of our process.

Given $\operatorname{road}(c)=\left(B_{\beta}\right)_{(\beta \sqsubseteq \alpha)}$ and $\operatorname{road}\left(c^{\prime}\right)=\left(B_{\beta}^{\prime}\right)_{\left(\beta \sqsubseteq \alpha^{\prime}\right)}$ for some $c \in C_{\alpha}$ and $c^{\prime} \in C_{\alpha^{\prime}}$, let us define $\operatorname{road}(\bar{c}) \triangleleft \operatorname{road}\left(c^{\prime}\right)$ if and only if $\alpha \sqsubset \alpha^{\prime}$ and $B_{\beta}=B_{\beta}^{\prime}$ for all $\beta \sqsubseteq \alpha$. Basically this is the prefix order for ordinal-sized sequences of connected components.

Now let $O=\{\operatorname{road}(c) \mid c \in A\}$. Note that $\mathcal{O}=\left(O, \triangleleft, \perp_{\triangleleft}\right)$ is an ordinal forest, because for each $c \in C_{\alpha}$ the order $\left(\left\{\operatorname{road}\left(c^{\prime}\right) \mid \operatorname{road}\left(c^{\prime}\right) \unlhd \operatorname{road}(c)\right\}, \unlhd\right)$ forms the ordinal $\alpha$ (for each $\beta \sqsubset \alpha$ it contains exactly one road of length $\beta$ ).

Now we show that the mapping $h$ with $h(c)=\operatorname{road}(c)$ is a homomorphism from $\mathcal{A}$ to $\mathcal{O}$. Take elements $a, a^{\prime} \in A$ with $a \in C_{\alpha}$, and $a^{\prime} \in C_{\alpha^{\prime}}$ for some $\alpha, \alpha^{\prime} \sqsubset \chi$. Let $\operatorname{road}(a)=\left(B_{\beta}\right)_{(\beta \sqsubseteq \alpha)}$ and $\left.\operatorname{road}\left(a^{\prime}\right)=\left(B_{\beta}^{\prime}\right)_{\left(\beta \sqsubseteq \alpha^{\prime}\right.}\right)$.

- If $a<a^{\prime}$, then (i) $\alpha \sqsubset \alpha^{\prime}$, because $a^{\prime}$ cannot be central point of a set which contains $a$, and (ii) $B_{\beta}=B_{\beta}^{\prime}$ for all $\beta \sqsubseteq \alpha$ because $a$ and $a^{\prime}$ belong to the same connected component of $A \backslash C_{\sqsubset \beta}$ for all $\beta \sqsubseteq \alpha$. By these observations we deduce that $\operatorname{road}(a) \triangleleft \operatorname{road}\left(a^{\prime}\right)$.

- If $a \perp a^{\prime}$, then, without loss of generality, suppose that $\alpha \sqsubseteq \alpha^{\prime}$. At stage $\alpha, a$ is a central point of $B_{\alpha} \in \mathcal{C}_{\alpha}$. Since $\alpha \sqsubseteq \alpha^{\prime}$, the connected component $B_{\alpha}^{\prime}$ exists. We must have $B_{\alpha} \neq B_{\alpha}^{\prime}$, since otherwise we would have $a \perp a^{\prime} \in$ $B_{\alpha}$ contradicting the fact that $a$ is central for $B_{\alpha}$. Therefore, $\operatorname{road}(a) \perp_{\triangleleft}$ $\operatorname{road}\left(a^{\prime}\right)$.

We finally add one extra element road $_{0}$ and make this the minimal element of $\mathcal{O}$, thus finding a homomorphism from $\mathcal{A}$ into an ordinal tree.

We can now complete the proof of the second part of Theorem 2

Proposition 18. The class $\Omega$ of all ordinal trees has EHD.

Proof. Given a $\{<, \perp\}$-structure $\mathcal{A}$, it suffices by Lemma 17 to find an MSOformula expressing the fact that every non-empty connected subset of $\mathcal{A}$ has a central point. Recall the WMSO-formula $\psi$ from Theorem 16. Seen as an MSOformula, $\psi$ clearly does the job. 
Remark 19. The procedure described in the proof of Lemma 17 can be also used to embed a structure $\mathcal{A}=(A,<, \perp)$ into an ordinary tree (where for every $x$, the set of all elements smaller than $x$ forms a finite linear order). For this, the ordinal $\chi$ has to satisfy $\chi \leq \omega$, i.e., every element $a \in A$ has to belong to a set $C_{n}$ for some finite $n$. We use this observation in Section 6 . Unfortunately, our results from Section 6 imply that $\chi \leq \omega$ cannot be expressed in Bool(MSO, WMSO+B).

\section{Constraint ECTL* over Trees of Height $h$}

Fix $h \in \mathbb{N}$. The aim of this section is to show that the class $\Theta_{h}$ of all trees of height $h$ (over $\{<, \perp\}$ ) has EHD. The proof relies on the fact that we can unfold the fixpoint procedure on the central points from the ordinal tree setting for $h$ steps in MSO.

For this section, we fix an arbitrary structure $\mathcal{A}=(A,<, \perp)$. We first define subsets $A_{0}, A_{1}, \ldots, A_{h} \subseteq A$ that are pairwise disjoint. The elements of $A_{0}$ are the central points of $A$ (this set is possibly empty) and, for each $i \geq 1, A_{i}$ contains the central points of each connected component of $A \backslash\left(A_{0} \cup \cdots \cup A_{i-1}\right)$. Note that $A_{0}$ contains exactly those nodes of $\mathcal{A}$ that a homomorphism from $\mathcal{A}$ to some tree can map to the root of the tree because elements from $A_{0}$ are neither incomparable to any other element nor below any other element, while all element outside of $A_{0}$ have to be incomparable to some other element or have to be below some other element. Hence they cannot be mapped to the root by any homomorphism. Thus, there is a homomorphism from $\mathcal{A}$ to some element of $\Theta_{h}$ if and only if $\mathcal{A} \backslash A_{0}$ can be embedded into some forest of height $h-1$. Now the sets $A_{i}$ for $1 \leq i \leq h$ collect exactly those elements which are chosen in the $i$-th step of the fixpoint procedure from the proof of Lemma 17 (where this set is called $C_{i}$ ). Thus, if $A_{0}, A_{1}, \ldots, A_{h}$ form a partition of $A$, then $\mathcal{A}$ allows a homomorphism to some $\mathcal{T} \in \Theta_{h}$. It turns out that the converse is also true. If $\mathcal{A} \preceq \mathcal{T}$ for some $\mathcal{T} \in \Theta_{h}$ then $A_{0}, A_{1}, \ldots, A_{h}$ form a partition of $A$. Thus, it suffices to show that each $A_{i}$ is MSO-definable. To do this, we define for all $i \in \mathbb{N}$ the formulas

$$
\begin{aligned}
\varphi_{0}(x) & :=\forall y \neg(y<x \vee y \perp x \vee x \perp y), \\
\varphi_{i+1}(x) & :=\forall y\left(\operatorname{con}_{i+1}(x, y) \rightarrow \neg(y<x \vee y \perp x \vee x \perp y)\right), \\
\operatorname{con}_{i+1}(x, y) & :=\exists Z \forall z\left(z \in Z \rightarrow \bigwedge_{j=0}^{i} \neg \varphi_{j}(z)\right) \wedge \operatorname{reach}(x, y, Z),
\end{aligned}
$$

where reach $(x, z, Z)$ is defined as in the proof of Proposition 16 on page 13, Let $A_{0}$ be the set of nodes $a \in A$ such that $\mathcal{A} \models \varphi_{0}(a)$ and let $A_{i+1}$ be the set of nodes $a \in A$ such that $\mathcal{A} \models \varphi_{i+1}(a)$.

Clearly $A_{0}$ is the set of central points of $A$. Inductively, one shows that $A_{i+1}$ is the set of central points of the connected components of $A \backslash\left(A_{0} \cup \cdots \cup A_{i}\right)$.

Lemma 20. There exists $\mathcal{T} \in \Theta_{h}$ such that $\mathcal{A} \preceq \mathcal{T}$ if and only if $A_{0}, A_{1}, \ldots, A_{h}$ is a partition of $A$. 
Proof. For the direction $(\Rightarrow)$ take a homomorphism $g$ from $\mathcal{A}$ to a tree $\mathcal{T}=$ $\left(T, \triangleleft, \perp_{\triangleleft}\right) \in \Theta_{h}$. By induction we prove that if $g$ maps $a$ to the $i$-th level of $\mathcal{T}$ then $a \in A_{j}$ for some $j \leq i$. For $i=0$ assume that $g(a)$ is the root of the tree. Then $a$ cannot be incomparable or greater than any other element. Thus, it is a central point of $A$, i.e., $a \in A_{0}$.

For the inductive step, assume that $g(a)$ is on the $i$-th level for $i>0$. Heading for a contradiction, assume that $a$ is neither in $A_{0} \cup \cdots \cup A_{i-1}$ nor a central point of some connected component of $A \backslash\left(A_{0} \cup \cdots \cup A_{i-1}\right)$. Then there is some $a^{\prime} \in A \backslash\left(A_{0} \cup \cdots \cup A_{i-1}\right)$ such that $a$ and $a^{\prime}$ are in the same connected component of $A \backslash\left(A_{0} \cup \cdots \cup A_{i-1}\right)$ and one of $a^{\prime}<a, a^{\prime} \perp a$ or $a \perp a^{\prime}$ holds. Since $g$ is a homomorphism, we get $g\left(a^{\prime}\right) \triangleleft g(a)$ or $g\left(a^{\prime}\right) \perp_{\triangleleft} g(a)$. If $g\left(a^{\prime}\right) \triangleleft g(a)$, then $a^{\prime}$ has to be mapped by $g$ to some level $j<i$, whence $a^{\prime} \in A_{0} \cup \cdots \cup A_{j}$ by the inductive hypothesis. This contradicts our assumption on $a^{\prime}$. Now, assume that $g\left(a^{\prime}\right) \perp_{\triangleleft} g(a)$. Let $a=a_{0}, a_{1}, \ldots, a_{m}=a^{\prime}$ be a path connecting $a$ and $a^{\prime}$ in $A \backslash\left(A_{0} \cup \cdots \cup A_{i-1}\right)$. Since $a_{i} \notin A_{0} \cup \cdots \cup A_{i-1}$, the inductive hypothesis shows that all $g\left(a_{i}\right)$ are on level $i$ or larger. But then, since $a_{0}, a_{1}, \ldots, a_{m}$ is a path, all $g\left(a_{i}\right)$ must belong to the subtree rooted at $g(a)$. This leads to the contradictions that $g\left(a_{m}\right)=g\left(a^{\prime}\right)$ is in the subtree rooted at $g(a)$ and hence is not incomparable to $g(a)$. Thus, we can conclude that $a \in A_{0} \cup \cdots \cup A_{i}$.

For the direction $(\Leftarrow)$ assume that $A_{0} \cup \cdots \cup A_{h}=A$. Applying the same construction described in the proof of Lemma 17 for ordinal trees, it is not hard to see that we find a homomorphism $g$ from $\mathcal{A}$ to some tree of height $h$ which maps the elements of $A_{i}$ to elements on level $i$. Should $A_{0}$ be empty, then $A$ would not be connected, and we would have a forest of height $h-1$. Adding a minimal element we still get a tree of height $h$.

Theorem 21. $\Theta_{h}$ has EHD.

Proof. Let $\mathcal{A}$ be any $\{<, \perp\}$-structure. Then, by Lemma 20, $\mathcal{A} \preceq \mathcal{T}$ for some $\mathcal{T} \in \Theta_{h}$ if and only if

$$
\mathcal{A} \models \forall x \bigvee_{i=0}^{h} \varphi_{i}(x)
$$

\section{Trees do not have EHD}

Let $\Theta$ be the class of all countable trees (over $\{<, \perp\}$ ). In this section, we prove that the logic Bool(MSO,WMSO+B) (the most expressive logic for which the EHD-technique currently works) cannot distinguish between graphs that admit a homomorphism to some element of $\Theta$ and those that do not. Heading for a contradiction, assume that $\varphi$ is a sentence such that a countable structure $\mathcal{A}=(A,<, \perp)$ satisfies $\varphi$ if and only if there is a homomorphism from $\mathcal{A}$ to some $\mathcal{T} \in \Theta$. Let $k$ be the quantifier rank of $\varphi$. We construct two graphs $\mathcal{E}_{k}$ and $\mathcal{U}_{k}$ such that $\mathcal{E}_{k}$ admits a homomorphism into a tree while $\mathcal{U}_{k}$ does not. We then use an Ehrenfeucht-Fraïssé game for Bool(MSO, WMSO+B) to show that $\varphi$ cannot separate these two structures, contradicting our assumption. This contradiction shows that $\Theta$ does not have EHD proving our second main result Theorem 1 . 


\subsection{The WMSO+B-Ehrenfeucht-Fraïssé-game}

The $k$-round WMSO+B-EF-game on a pair of structures $(\mathcal{A}, \mathcal{B})$ over the same finite relational signature $\sigma$ is played by spoiler and duplicator as follows 6 In the following, $A$ denotes the domain of $\mathcal{A}$ and $B$ the domain of $\mathcal{B}$.

The game starts in position

$$
p_{0}:=(\mathcal{A}, \emptyset, \emptyset, \mathcal{B}, \emptyset, \emptyset) .
$$

In general, before playing the $i$-th round (for $1 \leq i \leq k$ ) the game is in a position

$$
p=\left(\mathcal{A}, a_{1}, a_{2}, \ldots, a_{i_{1}}, A_{1}, A_{2}, \ldots, A_{i_{2}}, \mathcal{B}, b_{1}, b_{2}, \ldots, b_{i_{1}}, B_{1}, B_{2}, \ldots, B_{i_{2}}\right),
$$

where

1. $i_{1}, i_{2} \in \mathbb{N}$ satisfy $i_{1}+i_{2}=i-1$,

2. $a_{j} \in A$ for all $1 \leq j \leq i_{1}$,

3. $b_{j} \in B$ for all $1 \leq j \leq i_{1}$,

4. $A_{j} \subseteq A$ is a finite set for all $1 \leq j \leq i_{2}$, and

5. $B_{j} \subseteq B$ is a finite set for all $1 \leq j \leq i_{2}$.

In the $i$-th round spoiler and duplicator produce the next position as follows. Spoiler chooses to play one of the following three possibilities:

1. Spoiler can play an element move. For this he chooses either some $a_{i_{1}+1} \in A$ or $b_{i_{1}+1} \in B$. Duplicator then responds with an element from the other structure, i.e., with $b_{i_{1}+1} \in B$ or $a_{i_{1}+1} \in A$. The position in the next round is

$$
\left(\mathcal{A}, a_{1}, a_{2}, \ldots, a_{i_{1}+1}, A_{1}, A_{2}, \ldots, A_{i_{2}}, \mathcal{B}, b_{1}, b_{2}, \ldots, b_{i_{1}+1}, B_{1}, B_{2}, \ldots, B_{i_{2}}\right) .
$$

2. Spoiler can play a set move. For this he chooses either some finite $A_{i_{2}+1} \subseteq A$ or some finite $B_{i_{2}+1} \subseteq B$. Duplicator then responds with a finite set from the other structure, i.e., with $B_{i_{2}+1} \subseteq B$ or $A_{i_{2}+1} \subseteq A$. The position in the next round is

$$
\left(\mathcal{A}, a_{1}, a_{2}, \ldots, a_{i_{1}}, A_{1}, A_{2}, \ldots, A_{i_{2}+1}, \mathcal{B}, b_{1}, b_{2}, \ldots, b_{i_{1}}, B_{1}, B_{2}, \ldots, B_{i_{2}+1}\right) .
$$

3. Spoiler can play a bound move. For this he chooses one of the structures $\mathcal{A}$ or $\mathcal{B}$ and chooses a natural number $l \in \mathbb{N}$. Duplicator responds with another number $m \in \mathbb{N}$. Then the game continues as in the case of a set move with the restrictions that spoiler has to choose a subset of size at least $m$ from his chosen structure and duplicator has to respond with a set of size at least $l$.

After $k$ rounds, the game ends in a position

$$
p=\left(\mathcal{A}, a_{1}, a_{2}, \ldots, a_{i_{1}}, A_{1}, A_{2}, \ldots, A_{i_{2}}, \mathcal{B}, b_{1}, b_{2}, \ldots, b_{i_{1}}, B_{1}, B_{2}, \ldots, B_{i_{2}}\right) .
$$

Duplicator wins the game if

\footnotetext{
${ }^{6}$ For the ease of presentation we assume that $\mathcal{A}$ and $\mathcal{B}$ are infinite structures.
} 
1. $a_{j} \in A_{k} \Leftrightarrow b_{j} \in B_{k}$ for all $1 \leq j \leq i_{1}$ and all $1 \leq k \leq i_{2}$,

2. $a_{j}=a_{k} \Leftrightarrow b_{j}=b_{k}$ for all $1 \leq j<k \leq i_{1}$, and

3. for all relation symbols $R \in \sigma$ (of arity $n$ ) $\left(a_{j_{1}}, a_{j_{2}}, \ldots, a_{j_{n}}\right) \in R^{\mathcal{A}} \Leftrightarrow$ $\left(b_{j_{1}}, b_{j_{2}}, \ldots, b_{j_{n}}\right) \in R^{\mathcal{B}}$ for all $j_{1}, j_{2}, \ldots, j_{n} \in\left\{1, \ldots, i_{1}\right\}$.

As one would expect, the WMSO+B-EF-game can be used to show undefinability results for $\mathrm{WMSO}+\mathrm{B}$ due to the relationship between winning strategies in the $k$-round game and equivalence with respect to formulas up to quantifier rank $k$.

Proposition 22. For given $\sigma$-structures $\mathcal{A}$ and $\mathcal{B}$, elements $a_{1}, a_{2}, \ldots, a_{i_{1}} \in \mathcal{A}$, $b_{1}, b_{2}, \ldots, b_{i_{1}} \in \mathcal{B}$ and finite sets $A_{1}, A_{2}, \ldots, A_{i_{2}} \subseteq \mathcal{A}, B_{1}, B_{2}, \ldots, B_{i_{2}} \subseteq \mathcal{B}$, define the position

$$
p=\left(\mathcal{A}, a_{1}, \ldots, a_{i_{1}}, A_{1}, \ldots, A_{i_{2}}, \mathcal{B}, b_{1}, \ldots, b_{i_{1}}, B_{1}, \ldots, B_{i_{2}}\right) .
$$

Then, $\left(\mathcal{A}, a_{1}, a_{2}, \ldots, a_{i_{1}}, A_{1}, A_{2}, \ldots, A_{i_{2}}\right)$ and $\left(\mathcal{B}, b_{1}, b_{2}, \ldots, b_{i_{1}}, B_{1}, B_{2}, \ldots, B_{i_{2}}\right)$ are indistinguishable by any WMSO+B-formula $\varphi\left(x_{1}, \ldots, x_{i_{1}}, X_{1}, \ldots, X_{i_{2}}\right)$ of quantifier rank $k$ if and only if duplicator has a winning strategy in the $k$-round WMSO+B-EF-game started in $p$.

Proof. First of all note that up to logical equivalence there are only finitely many different WMSO + B-formulas $\varphi\left(x_{1}, \ldots, x_{i_{1}}, X_{1}, \ldots, X_{i_{2}}\right)$ of quantifier rank $k$. This fact is proved in a completely analogous way to the case of first-order or monadic second-order logic.

The proof is by induction on $k$. The base case $k=0$ is trivial. Assume now that the proposition holds for $k-1$. We use the abbreviations $\bar{a}=\left(a_{1}, \ldots, a_{i_{1}}\right)$, $\bar{A}=\left(A_{1}, \ldots, A_{i_{2}}\right), \bar{b}=\left(b_{1}, \ldots, b_{i_{1}}\right)$, and $\bar{B}=\left(B_{1}, \ldots, B_{i_{2}}\right)$ in the following. First assume that there is a WMSO+B-formula $\varphi\left(x_{1}, \ldots, x_{i_{1}}, X_{1}, \ldots, X_{i_{2}}\right)$ of quantifier rank $k$ such that

$$
\mathcal{A} \models \varphi(\bar{a}, \bar{A})
$$

and

$$
\mathcal{B} \not \models \varphi(\bar{b}, \bar{B}) .
$$

We show that spoiler has a winning strategy in the $k$-round game by a case distinction on the structure of $\varphi$. We only consider the case $\varphi=\mathrm{B} X \psi$ (all other cases can be handled exactly as in the WMSO-EF-game, see e.g. [10]). Let $l \in \mathbb{N}$ be a strict bound witnessing (3), in the sense that there is no set $A_{i_{2}+1}$ of size at least $l$ such that $\mathcal{A} \models \varphi\left(\bar{a}, \bar{A}, A_{i_{2}+1}\right)$. Then spoiler chooses structure $\mathcal{B}$ and bound $l$. Duplicator responds with some bound $m \in \mathbb{N}$. Due to (4)

$$
\mathcal{B} \models \neg \mathrm{B} X \psi(\bar{b}, \bar{B}, X) .
$$

Hence, there is a set $B_{i_{2}+1}$ of size at least $m$ such that

$$
\mathcal{B} \models \psi\left(\bar{b}, \bar{B}, B_{i_{2}+1}\right) .
$$

Spoiler chooses this set $B_{i_{2}+1}$. Duplicator must answer with a set $A_{i_{2}+1}$ of size at least $l$. By the choice of $l$ we conclude that

$$
\mathcal{A} \not \models \psi\left(\bar{a}, \bar{A}, A_{i_{2}+1}\right) .
$$


By the inductive hypothesis, spoiler has a winning strategy in the resulting position for the $(k-1)$-round game.

For the other direction, assume that $(\mathcal{A}, \bar{a}, \bar{A})$ and $(\mathcal{B}, \bar{b}, \bar{B})$ are indistinguishable by WMSO + B-formulas of quantifier rank $k$. Duplicator's strategy is as follows.

- If spoiler plays an element move choosing without loss of generality $a_{i_{1}+1} \in$ $\mathcal{A}$, let $\Phi$ be the set of all WMSO+B-formulas $\varphi$ of quantifier rank $k-1$ such that $\mathcal{A} \models \varphi\left(\bar{a}, a_{i_{1}+1}, \bar{A}\right)$. Since $\Phi$ is finite up to logical equivalence, there is a WMSO+B-formula $\psi$ of quantifier rank $k-1$ such that $\psi \equiv \bigwedge_{\varphi \in \Phi} \varphi$. By the assumption (indistinguishability up to quantifier rank $k$ ) and the fact that $\mathcal{A} \models \exists x \psi(\bar{a}, x, \bar{A})$ we conclude that $\mathcal{B} \models \exists x \psi(\bar{b}, x, \bar{B})$. Hence, there is an element $b_{i_{1}+1} \in \mathcal{B}$ such that $\mathcal{B} \models \psi\left(\bar{b}, b_{i_{1}+1}, \bar{B}\right)$. Thus, duplicator can respond with $b_{i_{1}+1}$ and obtain a position for which he has a winning strategy by the induction hypothesis.

- If spoiler plays a set move, we use the same strategy as in the element move. We only have to replace the element $a_{i_{1}+1}$ by Spoiler's set $A_{i_{1}+1}$ and the first-order quantifier by a set quantifier.

- Assume that spoiler plays a bound move, choosing $\mathcal{B}$ and bound $l \in \mathbb{N}$. Let

$$
\Phi_{A}=\{\varphi \mid \operatorname{rank}(\varphi)=k-1, \forall M \subseteq \mathcal{A}(|M| \geq l \Rightarrow \mathcal{A} \not \models \varphi(\bar{a}, \bar{A}, M))\} .
$$

Note that $\mathcal{A} \models \mathrm{B} X \varphi(\bar{a}, \bar{A}, X)$ for all $\varphi \in \Phi_{A}$. Thus, $\mathcal{B} \models \mathrm{B} X \varphi(\bar{b}, \bar{B}, X)$ for all $\varphi \in \Phi_{A}$. Since $\Phi_{A}$ is finite up to equivalence we can fix a number $m \in \mathbb{N}$ that serves as a bound in $(\mathcal{B}, \bar{b}, \bar{B})$ for all $\varphi \in \Phi_{A}$. Thus, for the set

$$
\Phi_{B}=\{\varphi \mid \operatorname{rank}(\varphi)=k-1, \forall M \subseteq \mathcal{B}(|M| \geq m \Rightarrow \mathcal{B} \not \models \varphi(\bar{b}, \bar{B}, M))\}
$$

we have $\Phi_{A} \subseteq \Phi_{B}$. Duplicator answers Spoiler's challenge with this number $m$. Then spoiler has to choose a set $B_{i_{2}+1} \subseteq \mathcal{B}$ of size at least $m$. Let

$$
\Psi_{B}=\left\{\varphi \mid \operatorname{rank}(\varphi)=k-1, \mathcal{B} \models \varphi\left(\bar{b}, \bar{B}, B_{i_{2}+1}\right)\right\} .
$$

Note that $\Phi_{B} \cap \Psi_{B}=\emptyset$. Since $\Psi_{B}$ is finite up to equivalence, there is a WMSO + B-formula $\psi \in \Psi_{B}$ of quantifier rank $k-1$ such that $\psi \equiv \bigwedge_{\varphi \in \Psi_{B}} \varphi$. In particular, $\psi \notin \Phi_{B}$. Hence, $\psi \notin \Phi_{A}$ (since $\Phi_{A} \subseteq \Phi_{B}$ ). By the definition of $\Phi_{A}$ this means that there is a subset $A_{i_{2}+1} \subseteq \mathcal{A}$ such that $\left|A_{i_{2}+1}\right| \geq l$ and $\mathcal{A} \models \psi\left(\bar{a}, \bar{A}, A_{i_{2}+1}\right)$. Duplicator chooses this set $A_{i_{2}+1}$. The resulting position allows a winning strategy for duplicator by the induction hypothesis.

\subsection{The Embeddable and the Unembeddable Triple-U-Structures}

In this section we define a class of finite structures $\mathcal{G}_{n, m}$ for $n, m \in \mathbb{N}$. Using these structures, we define for every $k \geq 0$ structures $\mathcal{E}_{k}$ and $\mathcal{U}_{k}$. We show that for every $k \geq 0, \mathcal{E}_{k}$ can be mapped homomorphically into a tree, whereas $\mathcal{U}_{k}$ cannot. In the next section, we will show that duplicator wins the $k$-round EF-game for both WMSO+B and MSO. 


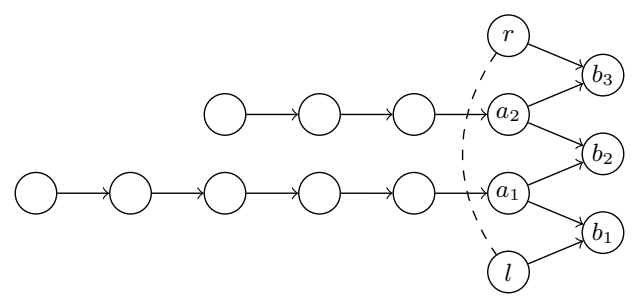

Fig. 2. The standard (5,3)-triple-u, where we only draw the Hasse diagram for $<^{D}$, and where dashed edges are $\perp$-edges.

The standard plain triple- $u$ is the structure $\mathcal{P}=\left(P,<^{P}, \perp^{P}\right)$, where

$$
\begin{aligned}
P & =\left\{l, r, a_{1}, a_{2}, b_{1}, b_{2}, b_{3}\right\}, \\
<^{P} & =\left\{\left(l, b_{1}\right),\left(a_{1}, b_{1}\right),\left(a_{1}, b_{2}\right),\left(a_{2}, b_{2}\right),\left(a_{2}, b_{3}\right),\left(r, b_{3}\right)\right\}, \text { and } \\
\perp^{P} & =\{(l, r),(r, l)\} .
\end{aligned}
$$

We call a structure $(V,<, \perp)$ a plain triple- $u$ if it is isomorphic to the standard plain triple-u. For $n, m \in \mathbb{N}$, the standard $(n, m)$-triple- $u$ is the structure $\mathcal{G}_{n, m}=$ $\left(D,<^{D}, \perp^{D}\right)$, where

$$
D=\left\{l, r, a_{1}, a_{2}, b_{1}, b_{2}, b_{3}\right\} \cup\left(\{1,2, \ldots, n\} \times\left\{a_{1}\right\}\right) \cup\left(\{1,2, \ldots, m\} \times\left\{a_{2}\right\}\right),
$$

and $<^{D}, \perp^{D}$ are the minimal relations such that

- $\mathcal{G}_{n, m}$ restricted to $\left\{l, r, a_{1}, a_{2}, b_{1}, b_{2}, b_{3}\right\}$ is the standard plain triple-u,

$-\left(a_{1}, 1\right)<\left(a_{1}, 2\right)<\cdots<\left(a_{1}, n\right)<a_{1}$, and

$-\left(a_{2}, 1\right)<\left(a_{2}, 2\right)<\cdots<\left(a_{2}, m\right)<a_{2}$.

We call a graph $(V,<, \perp)$ an $(n, m)$-triple- $u$ if it is isomorphic to the standard $(n, m)$-triple-u. Figure 2 depicts a $(5,3)$-triple-u.

Remark 23. For all $n, m \in \mathbb{N}$ and each $(n, m)$-triple-u $\mathcal{W}$ we fix an isomorphism $\psi_{\mathcal{W}}$ between $\mathcal{W}$ and the standard $(n, m)$-triple-u. Note that this isomorphism is unique if $n \neq m$. If $n=m$, then there is an automorphism of $\mathcal{G}_{n, n}$ exchanging the nodes $l$ and $r$. Thus, choosing an isomorphism means to choose the left node of the triple-u. For $x \in\left\{l, r, a_{1}, a_{2}, b_{1}, b_{2}, b_{3}\right\}$ we denote by $\mathcal{W} \cdot x$ the unique node $w \in \mathcal{W}$ such that $\psi_{\mathcal{W}}(w)=x$. Furthermore, we call the linear order of size $n$ (resp., $m$ ) that consists of all proper ancestors of $\psi_{\mathcal{W}}^{-1}\left(a_{1}\right)$ (resp., $\left.\psi_{\mathcal{W}}^{-1}\left(a_{2}\right)\right)$ the left order (resp., right order) of $\mathcal{W}$.

Let $k \in \mathbb{N}$ be a natural number. Fix a strictly increasing sequence $\left(n_{k, i}\right)_{i \in \mathbb{N}}$ such that the linear order of length $n_{k, i}$ and the linear order of length $n_{k, j}$ are equivalent with respect to WMSO+B-formulas of quantifier rank up to $k$ for all $i, j \in \mathbb{N}$. Such a sequence exists because there are (up to equivalence) only finitely many WMSO+B-formulas of quantifier rank $k$. Since the linear orders of length $n_{k, i}$ are finite, they are equivalent with respect to both MSO-formulas and WMSO-formulas of quantifier rank up to $k$. 
Definition 24 (The embeddable triple-u). Let $\mathcal{E}_{k}$ be the structure that consists of

1. the disjoint union of infinitely many $\left(n_{k, 1}, n_{k, j}\right)$-triple-u's and infinitely many $\left(n_{k, j}, n_{k, 1}\right)$-triple-u's for all $j \geq 2$,

2. one additional node $d$, and

3. for each triple-u $\mathcal{W}$ an <-edge from $\mathcal{W}$.l to $d$.

In the following we call $d$ the final node of $\mathcal{E}_{k}$

Lemma 25. For all $k \in \mathbb{N}, \mathcal{E}_{k}$ admits a homomorphism to a tree.

Proof. Using the procedure on the central points from the ordinal tree setting described in the proof of Lemma 17] we first start adding the chains of each triple- $u$ to the tree. In step $n_{k, 1}$ we finally have placed all the chains of length $n_{k, 1}$. Thus, for each triple-u $\mathcal{W}$ either $\mathcal{W} . a_{1}$ or $\mathcal{W} . a_{2}$ becomes central. Thus, in step $n_{k, 1}+1$ all the triple-u's split into two disconnected components and the incomparability edges, which were avoiding that $\mathcal{W} . l$ became central, now cease having such an effect. We can therefore map $\mathcal{W} . l$ at stage $n_{k, 1}+2$ and the final node $d$ in step $n_{k, 1}+3$. Thus, it is easy to prove that the fixpoint procedure from the proof of Lemma 17 terminates at stage $\omega$. Whenever this happens, the given structure admits a homomorphism to a tree, see Remark 19,

Definition 26 (The unembeddable triple-u). Let $\mathcal{U}_{k}$ be the structure that consists of

1. the disjoint union of infinitely many $\left(n_{k, j}, n_{k, j}\right)$-triple-u's for all $j \geq 2$,

2. one additional node $d$, and

3. for each triple-u $\mathcal{W}$ an <-edge from W.l to $d$.

In the following we call $d$ the final node of $\mathcal{U}_{k}$

Lemma 27. For all $k \in \mathbb{N}, \mathcal{U}_{k}$ does not admit a homomorphism to a tree.

Proof. Again, we consider the fixpoint procedure from the proof of Lemma 17. Assume that $\mathcal{U}_{k}$ admits a homomorphism to a tree. Then, the final node $d$ has to be placed at some stage $i$ into the tree, i.e., in the notation of the proof of Lemma 17] $d$ belongs to some set $C_{i}$ for $i<\omega$. But there is a $\left(n_{k, i}, n_{k, i}\right)$-triple-u $\mathcal{W}$ and $\mathcal{W} . l<d$. Hence, $\mathcal{W} . l$ has to be placed into the tree in one of the first $i-1$ stages. But $\mathcal{W} \cdot a_{1}$ and $\mathcal{W} \cdot a_{2}$ are the target nodes of chains of length $n_{k, i} \geq i$. Hence, after $i$ stages they are still not mapped into the tree. Therefore, after $i$ stages, $\mathcal{W} . l$ and $\mathcal{W} . r$ are in the same connected component and they are linked by an $\perp$-edge. This contradicts the fact that $\mathcal{W} . l$ was placed into the tree in one of the first $i-1$ stages. 


\subsection{Duplicators Strategies in the $k$-Round Game}

We show that $\Theta$ does not have EHD by showing that duplicator wins the $k$-round MSO-EF-game and WMSO+B-EF-game on the pair $\left(\mathcal{E}_{k}, \mathcal{U}_{k}\right)$ for each $k \in \mathbb{N}$. Hence, the two structures are not distinguishable by Bool(MSO, WMSO+B)formulas of quantifier rank $k$. For MSO this is rather simple. Since the linear orders of length $n_{k, i}$ and $n_{k, j}$ are indistinguishable up to quantifier rank $k$, it is straightforward to compile the strategies on these pairs of paths into a strategy on the whole structures for the $k$-round game. It is basically the same proof as the one showing that a strategy on a pair $\left(\biguplus_{i \in I} \mathcal{A}_{i}, \biguplus_{i \in I} \mathcal{B}_{i}\right)$ of disjoint unions can be compiled from strategies on the pairs $\left(\mathcal{A}_{i}, \mathcal{B}_{i}\right)$. In our situation there is an $i \in I$ such that $\mathcal{A}_{i}=\mathcal{B}_{i}$ consists of infinitely many plain triple-u's together with the final node, and the other pairs $\left(\mathcal{A}_{j}, \mathcal{B}_{j}\right)$ for $j \in I \backslash\{i\}$ consist of two linear orders that are indistinguishable by MSO-formulas of quantifier rank $k$. We leave the proof details as an exercise for the interested reader.

Compiling local strategies to a global strategy in the WMSO+B-EF-game is much more difficult because strategies are not closed under infinite disjoint unions. For instance, let $\mathcal{A}$ be the disjoint union of infinitely many copies of the linear order of size $n_{k, 1}$ and $\mathcal{B}$ be the disjoint union of all linear orders of size $n_{k, j}$ for all $j \in \mathbb{N}$. Clearly, duplicator has a winning strategy in the $k$-round game starting on the pair that consists of the linear order of size $n_{k, 1}$ and the linear order of size $n_{k, j}$. But in $\mathcal{A}$ every linear suborder has size bounded by $n_{k, 1}$, while $\mathcal{B}$ has linear suborders of arbitrary finite size. This difference is of course expressible in WMSO+B. Even though strategies in WMSO+B-games are not closed under disjoint unions, we can obtain a composition result for disjoint unions on certain restricted structures as follows. Let $\mathcal{A}=\biguplus_{i \in \mathbb{N}} \mathcal{A}_{i}$ and $\mathcal{B}=\biguplus_{i \in \mathbb{N}} \mathcal{B}_{i}$ be disjoint unions of structures $\mathcal{A}_{i}$ and $\mathcal{B}_{i}$ satisfying the following conditions:

1. All $\mathcal{A}_{i}$ and $\mathcal{B}_{i}$ are finite structures.

2. For every $i \in \mathbb{N}$, duplicator has a winning strategy in the $k$-round MSO-EFgame on $\mathcal{A}_{i}$ and $\mathcal{B}_{i}$.

3. There is a constant $c \in \mathbb{N} \backslash\{0\}$ such that whenever spoiler starts the MSOEF-game on $\left(\mathcal{A}_{i}, \mathcal{B}_{i}\right)$ with a set move choosing a set of size $n$ in $\mathcal{A}_{i}$ or $\mathcal{B}_{i}$, then duplicator's strategy answers with a set of size at least $\frac{n}{c}$.

In this case duplicator has a winning strategy in the $k$-round WMSO+B-EFgame on $\mathcal{A}$ and $\mathcal{B}$. To substantiate this claim, we sketch his strategy. For an element or set move, duplicator just uses the local strategies from the MSOgame to give an answer to any challenge. For a bound move, duplicator does the following. If spoiler's chooses the bound $l \in \mathbb{N}$, then duplicator chooses the number $m$, which is the total number of elements in all substructures $\mathcal{A}_{i}$ or $\mathcal{B}_{i}$ in which some elements have been chosen in one of the previous rounds plus $c \cdot l$. This forces spoiler to choose $c \cdot l$ elements in fresh substructures. Then duplicator uses his strategy in each local pair of structures to give an answer to spoiler's challenge. Since spoiler chose $c \cdot l$ elements in fresh substructures, duplicator answers with at least $\frac{c \cdot l}{c}=l$ many elements in fresh substructures. 
This is a valid move and it preserves the existence of local winning strategies between each pair $\left(\mathcal{A}_{i}, \mathcal{B}_{i}\right)$ for the rounds yet to play.

From now on, we consider a fixed number $k \in \mathbb{N}$ and the game on the structures $\mathcal{E}_{k}$ and $\mathcal{U}_{k}$. We use a variant of the closure under restricted disjoint unions, sketched above, to provide a winning strategy for duplicator. In order to reduce notational complexity we just write $\mathcal{E}$ for $\mathcal{E}_{k}, \mathcal{U}$ for $\mathcal{U}_{k}$ and $n_{i}$ for $n_{k, i}$ (for all $i \in \mathbb{N}$ ). With $\bar{E}$ (resp. $\bar{U}$ ) we denote the set of all maximal subgraphs that are $(n, m)$-triple-u's occurring in $\mathcal{E}$ (resp., $\mathcal{U}$ ) where $n$ and $m$ range over $\mathbb{N}$. Note that $\mathcal{E}$ is the disjoint union of all $W \in \bar{E}$ together with the final node, and similarly of $\mathcal{U}$. Unfortunately, we cannot apply the result on restricted disjoint unions directly because of the following problems.

- Due to the final nodes of $\mathcal{E}$ and $\mathcal{U}$, the structures are not disjoint unions of triple-u's. But since the additional structure in both structures is added in a uniform way this does not pose a problem for the proof.

- The greater cause for trouble is that there is no constant $c$ as in condition 3 that applies uniformly to all MSO-EF-games on an $\left(n_{j}, n_{1}\right)$-triple-u of $\mathcal{E}$ and an $\left(n_{j}, n_{j}\right)$-triple-u of $\mathcal{U}$ for all $j \in \mathbb{N}$. The problem is that if spoiler chooses in his first move all elements of the right order of the $\left(n_{j}, n_{j}\right)$-triple- $\mathrm{u}$, then the only possible answer of duplicator is to choose the set of the $n_{1}$ many elements of the right order of the $\left(n_{j}, n_{1}\right)$-triple-u. But since the numbers $n_{j}$ grow unboundedly, there is not constant $c$ such that the inequation $n_{1} \geq c n_{j}$ holds for all $j$. This problem does not exist for moves where spoiler chooses many elements in the left order of the $\left(n_{j}, n_{j}\right)$-triple-u. Duplicator's strategy allows to exactly choose the same subset of the left order of the $\left(n_{j}, n_{1}\right)$ triple-u. This allows to overcome the problem that duplicator should answer challenges where spoiler chooses a large set with an equally large set (up to some constant factor): Instead of assigning each triple-u in $\bar{E}$ a fixed corresponding triple-u in $\bar{U}$, we do this dynamically. If spoiler chooses a lot of elements from the left order of a fresh $\left(n_{j}, n_{j}\right)$-triple-u, then duplicator answers this challenge in a $\left(n_{j}, n_{1}\right)$-triple- $\mathrm{u}$ and we consider these two structures as forming one pair of the disjoint unions. On the other hand, if spoiler chooses a lot of elements from the right order of a fresh $\left(n_{j}, n_{j}\right)$-triple-u, then duplicators corresponding structure is chosen to be a fresh $\left(n_{1}, n_{j}\right)$-triple-u. In any case duplicator's local winning strategy may copy most of spoiler's choice (i.e., all elements chosen from the plain triple-u and from the order of length $n_{j}$ from which spoiler has chosen more elements), thus producing a set which is at least half as big as spoiler's challenge.

In our prove we encode this dynamic choice of corresponding structures as a partial map $\varphi: \bar{E} \rightarrow \bar{U}$. The following definition of a locally-i-winning position describes the requirements on a position obtained after playing some rounds that allow to further use local winning strategies in order to compile a winning strategy for the next $i$-rounds. It basically requires that the map $\varphi$ is such that for each triple-u $W \in \operatorname{dom}(\varphi)$ the restriction of the current position to $W$ and $\varphi(W)$ is a valid position in the $i$-round WMSO+B-EF-game on $(W, \varphi(W))$ which 
is winning for duplicator and that $\operatorname{dom}(\varphi)$ and $\operatorname{im}(\varphi)$ covers all elements that have been chosen so far (in an element move or as a member of some set).

Definition 28. A position

$$
p=\left(\mathcal{E}, e_{1}, \ldots, e_{i_{1}}, E_{1}, \ldots, E_{i_{2}}, \mathcal{U}, u_{1}, \ldots, u_{i_{1}}, U_{1}, \ldots, U_{i_{2}}\right)
$$

in the $\mathrm{WMSO}+\mathrm{B}-E F$-game on $(\mathcal{E}, \mathcal{U})$ is called locally-i-winning (for duplicator) if there is a partial bijection $\varphi: \bar{E} \rightarrow \bar{U}$ such that

$-\operatorname{dom}(\varphi)$ is finite,

- for all $W \in \bar{E}, W^{\prime} \in \bar{U}$, and $1 \leq j \leq i_{1}$,

1. if $e_{j} \in W$ then $W \in \operatorname{dom}(\varphi)$ and $u_{j} \in \varphi(W)$, and

2. if $u_{j} \in W^{\prime}$ then $W^{\prime} \in \operatorname{im}(\varphi)$ and $e_{j} \in \varphi^{-1}\left(W^{\prime}\right)$,

- for all $W \in \bar{E}, W^{\prime} \in \bar{U}$, and $1 \leq j \leq i_{2}$,

1. if $E_{j} \cap W \neq \emptyset$ then $W \in \operatorname{dom}(\varphi)$ and

2. if $U_{j} \cap W^{\prime} \neq \emptyset$ then $W^{\prime} \in \operatorname{im}(\varphi)$, and

- $\varphi$ is compatible with local strategies in the following sense:

1. For all $W \in \operatorname{dom}(\varphi), x \in\left\{l, r, a_{1}, a_{2}, b_{1}, b_{2}, b_{3}\right\}, 1 \leq j \leq i_{1}$ and $1 \leq k \leq$ $i_{2}$ we have

- $e_{j}=W \cdot x \Leftrightarrow u_{j}=\varphi(W) \cdot x$, and

- $W . x \in E_{k} \Leftrightarrow \varphi(W) . x \in U_{k}$.

2. For all $W \in \operatorname{dom}(\varphi)$ and $1 \leq j \leq i_{1}, e_{j}$ belongs to the left (resp., right) order of $W$ if and only if $u_{j}$ belongs to the left (resp., right) order of $\varphi(W)$.

3. For each $W \in \operatorname{dom}(\varphi)$, the restriction of the position $p$ to the left (resp., right) order of $W$ and the left (resp., right) order of $\varphi(W)$ is a winning position for duplicator in the $i$-round WMSO-EF-game.

4. For all $1 \leq j \leq i_{1}, e_{j}$ is the final node of $\mathcal{E}$ if and only if $u_{j}$ is the final node of $\mathcal{U}$.

5. For all $1 \leq j \leq i_{2}, E_{j}$ contains the final node of $\mathcal{E}$ if and only if $U_{j}$ contains the final node of $\mathcal{U}$.

Remark 29. Note that the WMSO+B-EF-game on $(\mathcal{E}, \mathcal{U})$ starts in a locally$k$-winning position where the partial map $\varphi$ is the map with empty domain. Moreover, for all $i \in \mathbb{N}$, every locally- $i$-winning position is a winning position for duplicator in the 0-round WMSO+B-EF-game.

Proposition 30. Duplicator has a winning strategy in the $k$-round WMSO+BEF-game on $\left(\mathcal{E}_{k}, \mathcal{U}_{k}\right)$.

Due to the previous remark, the proposition follows directly form the following lemma.

Lemma 31. Let $1 \leq i \leq k$ be a natural number and $p$ a locally-i-winning position. Duplicator can respond any challenge of spoiler such that the next position is locally-(i-1)-winning. 
Proof. Let $\varphi: \bar{E} \rightarrow \bar{U}$ be the partial bijection for the locally- $i$-winning position $p$. In the following, we say that an $(n, m)$-triple-u is fresh if it does not belong to $\operatorname{dom}(\varphi) \cup \operatorname{im}(\varphi)$. We consider the three possible types of moves for spoiler.

1. If spoiler plays an element move, there are the following possibilities.

- If spoiler chooses the final node of one of the structures, duplicator answers with the final node of the other.

- If spoiler chooses some node from an $(n, m)$-triple-u $W \in \operatorname{dom}(\varphi)$, then the local strategies allow duplicator to answer this move with a node from $\varphi(W)$.

- Analogously, if spoiler chooses some node from an $(n, m)$ - triple-u $W \in$ $\operatorname{im}(\varphi)$, then the local strategies allow duplicator to answer this move with a node from $\varphi^{-1}(W)$.

- If spoiler chooses a node from a fresh $(n, m)$-triple-u $W$ then duplicator can choose some fresh $\left(n^{\prime}, m^{\prime}\right)$-triple-u $W^{\prime}$ from the other structure and can use the WMSO-equivalence up to quantifier rank $k$ of the left and right orders of $W$ and $W^{\prime}$ to find a response to spoilers challenge such that adding $\left(W, W^{\prime}\right)$ (or $\left(W^{\prime}, W\right)$ depending on whether $\left.W \in \bar{E}\right)$ to $\varphi$ leads to a locally- $(i-1)$-winning position.

2 . If spoiler plays a set move, then he chooses a finite set containing elements from some of the triple-u's from $\operatorname{dom}(\varphi)$ or $\operatorname{im}(\varphi)$ and from $l$ many fresh triple-u's. Choosing $l$ fresh triple-u's from the other structure, we can find a response on each of the triple-u's corresponding to the local strategy similar to the case of the element move. The union of all these local responses is a response for duplicator that leads to a locally- $(i-1)$-winning position.

3 . If spoiler plays a bound move, we distinguish which structure he chooses.

- If he chooses structure $\mathcal{U}$ and the bound $l \in \mathbb{N}$, let $Z_{n}$ be the (finite) set of all $(n, n)$-triple-u's occurring in $\operatorname{im}(\varphi)$ and set

$$
m_{1}=\sum_{n \in \mathbb{N}} \sum_{W \in Z_{n}}(2 n+7) .
$$

Duplicator responds with the bound $m=m_{1}+2 l$. Note that $2 n+7$ is the size of an $(n, n)$-triple-u. Hence $m_{1}$ is the number of nodes in non-fresh triple-u's of $\mathcal{U}$. Assume that spoiler chooses some finite subset $S$ of $\mathcal{U}$ with $|S| \geq m$. We construct a subset $S^{\prime}$ in $\mathcal{E}$ such that the resulting position is locally- $(i-1)$-winning. Moreover, we guarantee that for any fresh triple-u $W \in \bar{U}$ such that $S \cap W \neq \emptyset$, duplicator's response $S^{\prime} \cap W^{\prime}$ in a corresponding fresh triple-u $W^{\prime} \in \bar{E}$ contains at least $\frac{1}{2}|S \cap W|$ many elements. If $W_{1}, \ldots, W_{z} \in \bar{U}$ are all the fresh triple-u's that intersect $S$ non trivially, then we already argued that $\left|\bigcup_{i=1}^{z}\left(W_{i} \cap S\right)\right| \geq m-m_{1}=2 l$. Hence, duplicator's response $S^{\prime}$ contains at least $l$ many elements as desired. The concrete choice of $S^{\prime}$ is done as follows.

(a) For all $W \in \operatorname{im}(\varphi)$, duplicator chooses a set $S_{W}^{\prime} \subseteq \varphi^{-1}(W)$ such that $S_{W}^{\prime}$ is the answer to Spoiler's challenge $S \cap W$ according to a winning strategy in the $i$-round WMSO-EF-game on the restriction of $p$ to $\varphi^{-1}(W)$ and $W$. This winning strategy exists because position $p$ is locally $i$-winning. 
(b) Now consider a fresh $(n, n)$-triple-u $W \in \bar{U}$ with $W \cap S \neq \emptyset$. Let $L$ (resp., $R$ ) be the nodes in the left (resp., right) order of $W$. If $|L \cap S| \geq|R \cap S|$, then take a fresh $\left(n, n_{1}\right)$-triple-u $W^{\prime} \in \bar{E}$ (note that $n \geq n_{1}$ ) and extend the partial bijection $\varphi$ by $\varphi\left(W^{\prime}\right)=W$. Duplicator chooses the subset $S_{W}^{\prime}=\psi(S \cap W \backslash R) \cup T$, where $\psi$ is the obvious isomorphism between the $(n, 0)$-sub-triple-u of $W$ (i.e., $W \backslash R)$ and the $(n, 0)$-sub-triple-u of $W^{\prime}$, and $T$ is an answer to spoilers move $S \cap R$ according to a winning strategy in the $i$-round WMSO-EF-game between the right order of $W^{\prime}$ and the right order of $W$. Note that $\left|S_{W}^{\prime}\right| \geq \frac{1}{2}|S \cap W|$.

If $|L \cap S|<|R \cap S|$, then let $W^{\prime}$ be an $\left(n_{1}, n\right)$ triple-u and use the same strategy but reverse the roles of the left and the right order of the chosen triple-u's.

(c) If the final node of $\mathcal{U}$ is in $S$, let $S_{d}^{\prime}$ be the singleton containing the final node of $\mathcal{E}$, otherwise let $S_{d}^{\prime}=\emptyset$.

Finally, let $S^{\prime}$ be the union of $S_{d}^{\prime}$ and all sets $S_{W}^{\prime}$ defined in (a) and (b) above. Since spoiler has chosen at least $2 l-1$ many elements from fresh triple-u's, we directly conclude that $\left|S^{\prime}\right| \geq l$. Moreover, since all the parts of $S^{\prime}$ were defined using local strategies, we easily conclude that the position reached by choosing $S^{\prime}$ is locally- $(i-1)$-winning.

- If spoiler chooses structure $\mathcal{E}$ and bound $l \in \mathbb{N}$, we use a similar strategy. Let $Y_{n}$ be the set of all $\left(n_{1}, n\right)$-triple-u's and all $\left(n, n_{1}\right)$-triple-u's occurring in $\operatorname{dom}(\varphi)$, and define

$$
m_{1}=\sum_{n \in \mathbb{N}} \sum_{W \in Y_{n}} n_{1}+n+7
$$

and $m_{2}=l \cdot n_{1}$. Note that $m_{1}$ is the number of nodes from non-fresh triple-u's from $\mathcal{E}$. Duplicator responds with $m=m_{1}+m_{2}+l$. Let $S \subseteq \mathcal{E}$ be Spoiler's set with $|S| \geq m$. There are at least $m_{2}+l$ elements in $S$ chosen from fresh triple-u's $W_{1}, W_{2}, \ldots, W_{z} \in \bar{E}$. Either $z>l$ or spoiler has chosen at least $l$ elements from $W_{1} \cup W_{2} \cup \cdots \cup W_{z}$ that do not belong to the orders of length $n_{1}$ (which in total contain only $z \cdot n_{1} \leq l \cdot n_{1}=m_{2}$ many elements). Duplicator chooses his response $S^{\prime}$ in $\mathcal{U}$ as follows:

(a) For all $W \in \operatorname{dom}(\varphi)$, duplicator chooses a set $S_{W}^{\prime} \subseteq \varphi(W)$ such that $S_{W}^{\prime}$ is the answer to Spoiler's challenge $S \cap W$ according to a winning strategy in the $i$-round WMSO-EF-game on the restriction of $p$ to $W$ and $\varphi(W)$. This winning strategy exists because position $p$ is locally $i$-winning.

(b) Now consider a fresh triple-u $W \in \bar{E}$ with $W \cap S \neq \emptyset$. If $W$ is an $\left(n_{1}, n\right)$-triple-u or an $\left(n, n_{1}\right)$-triple-u, let $W^{\prime} \in \bar{U}$ be a fresh $(n, n)$ triple-u of $\mathcal{U}$, and extend the partial bijection $\varphi$ by $\varphi(W)=W^{\prime}$. Let us consider the case that $W$ is an $\left(n, n_{1}\right)$-triple-u (for the other case one can argue analogously) and let $R$ be the right order (of size $n_{1}$ ) of $W$. Duplicator chooses the subset $S_{W}^{\prime}=\psi(S \cap W \backslash R) \cup T$, where $\psi$ is the obvious isomorphism between the $(n, 0)$-sub-triple-u of $W$ 
(i.e., $W \backslash R)$ and the $(n, 0)$-sub-triple-u of $W^{\prime}$, and $T$ is an answer to Spoiler's move $S \cap R$ according to a winning strategy in the $i$-round WMSO-EF-game between the right order of $W$ and the right order of $W^{\prime}$. We can assume that $S_{W}^{\prime} \neq \emptyset$. because we have $S \cap W \backslash R \neq \emptyset$ or $S \cap R \neq \emptyset$ and in the latter case $T$ can be chosen to be non-empty.

(c) If the final node of $\mathcal{E}$ is in $S$, let $S_{d}^{\prime}$ be the singleton containing the final node of $\mathcal{U}$, otherwise let $S_{d}^{\prime}=\emptyset$.

Finally, let duplicator's response $S^{\prime}$ be the union of $S_{d}^{\prime}$ and all sets $S_{W}^{\prime}$ defined in (a) and (b) above. By the argument before (a), duplicator selects in (b) in total at least $l$ elements. Moreover, since all the parts of $S^{\prime}$ where defined using local strategies, we easily conclude that the position reached by choosing $S^{\prime}$ is locally- $(i-1)$-winning.

\section{Open Problems}

The main open problem that remains is whether the problem $\operatorname{SAT-ECTL}{ }^{*}\left(\Gamma^{=}\right)$ is decidable for the class $\Gamma$ of all trees (or equivalently, the single infinite binary tree). We have only proved that the EHD-method cannot yield decidability. Currently, we are investigating automata theoretic approaches to this question.

\section{Acknowledgment}

We thank Manfred Droste for fruitful discussions on universal structures and semi-linear orders.

\section{References}

1. M. Bojańczyk and S. Toruńczyk. Weak MSO+U over infinite trees. In Proc. STACS 2012, vol. 14 of LIPIcs, 648-660. Schloss Dagstuhl - Leibniz-Zentrum für Informatik, 2012.

2. M. Bojańczyk and S. Toruńczyk. Weak MSO+U over infinite trees (long version). available at http://www.mimuw.edu.pl/ bojan/papers/wmsou-trees.pdf

3. M. Bojanczyk, T. Gogacz, H. Michalewski, and M. Skrzypczak. On the decidability of MSO+U on infinite trees. In Proc. ICALP (2) 2014, LNCS 8573, 50-61. Springer, 2014.

4. L. Bozzelli and R. Gascon. Branching-time temporal logic extended with qualitative Presburger constraints. In Proc. LPAR 2006, LNCS 4246, 197-211. Springer, 2006.

5. L. Bozzelli and S. Pinchinat Verification of gap-order constraint abstractions of counter systems. Theoretical Computer Science, 523:1-36, 2014.

6. C. Carapelle, A. Kartzow, and M. Lohrey. Satisfiability of CTL* with constraints. In Proc. CONCUR 2013, LNCS 8052, pages 455-469. Springer, 2013.

7. C. Carapelle, A. Kartzow and M. Lohrey. Satisfiability of ECTL $^{*}$ with constraints. submitted for publication. available at http://www .informatik.uni-leipzig.de/ kartzow/Paper/ECTL-With-Constraints.pdf 
8. S. Demri and R. Gascon. Verification of qualitative $\mathbb{Z}$ constraints. Theoretical Computer Science, 409:24-40, 2008.

9. M. Droste Structure of partially ordered sets with transitive automorphism groups Memoirs of the American Mathematical Society, 334, 1985

10. H.D. Ebbinghaus and J. Flum. Finite Model Theory. Perspectives in Mathematical Logic Series, Springer, 1995.

11. R. Gascon. An automata-based approach for $\mathrm{CTL}^{*}$ with constraints. Electronic Notes in Theoretical Computer Science, 239:193-211, 2009.

12. W. H. Gottschalk Choice functions and Tychonoff's theorem. Proceedings of the American Mathematical Society, 2:172, 1951.

13. R. Rado. Axiomatic treatment of rank in infinite sets. Canadian Journal of Mathematics 1:337-343, 1949.

14. W. Thomas. Computation tree logic and regular omega-languages. In Proc. REX Workshop 1988, LNCS 354, 690-713. Springer, 1988.

15. M. Y. Vardi and P. Wolper. Yet another process logic (preliminary version). In Proceedings of Logics of Programs 1983, LNCS 164, 501-512. Springer, 1983.

16. E. S. Wolk. The Comparability Graph of a Tree. Proceedings of the American Mathematical Society, 13(5):789-795, 1962.

17. E. S. Wolk. A Note on "The Comparability Graph of a Tree". Proceedings of the American Mathematical Society, 16)(1):17-20, 1965.

\section{A Universal Semi-linear Order}

In the following we define a semi-linear order which is universal for the class of all countable semi-linear orders. The fact that this order is universal is known to the experts in the field of semi-linear orders. Unfortunately, to our best knowledge there is no proof of this fact in the literature. Hence we provide a proof for this result.

Definition 32. Let $\mathcal{U}=(U,<, \perp)$ be the countable semi-linear order with:

$-U=(\mathbb{N Q})^{*}$,

$-<$ is the strict order induced by $n_{1} p_{1} n_{2} p_{2} \cdots n_{k} p_{k} \leq m_{1} q_{1} m_{2} q_{2} \cdots m_{l} q_{l}$ iff

- $k \leq l, n_{i}=m_{i}$ for all $1 \leq i \leq k, p_{i}=q_{i}$ for all $1 \leq i \leq k-1$ and $p_{k} \leq q_{k}$, and

- $\perp=\perp_{<}$.

$W e$ call $\mathcal{U}$ the universal countable semi-linear order.

Note that Droste [9] has already studied this and similar orders.

For $u=n_{1} p_{1} n_{2} p_{2} \cdots n_{k} p_{k} \in U$ (with $k \geq 1$ ) and $q \in \mathbb{Q}$, we define

$$
u+q=n_{1} p_{1} n_{2} p_{2} \cdots n_{k-1} p_{k-1} n_{k}\left(p_{k}+q\right) .
$$

We say that a countable semi-linear order $(A,<, \perp)$ is closed under finite infima if for each finite set $S \subseteq A$ the linear order $\{a \in A \mid a \leq s$ for all $s \in S\}$ has a maximal element, which is denoted by $\inf (S)$. Let $E=\left(a_{i}\right)_{i \in \mathbb{N}}$ be a repetitionfree enumeration of $A$. We say $E$ is closed under infima if for each initial subset $A_{i}=\left\{a_{1}, a_{2}, \ldots, a_{i}\right\}$ and each $S \subseteq A_{i}$ we have $\inf (S) \in A_{i}$. 
Lemma 33. Let $\mathcal{A}=(A,<, \perp)$ be a countable semi-linear order. There is a countable semi-linear order $\mathcal{B}$ that is closed under finite infima and an injective homomorphism from $\mathcal{A}$ to $\mathcal{B}$.

Proof. For a nonempty subset $S \subseteq A$ we set $\downarrow S=\{a \in A \mid \forall s \in S(a \leq s)\}$. Let $\bar{A}$ be the set of finite nonempty subsets of $A$, which is obviously countable. We define an equivalence on $\bar{A}$ by setting $S \sim T$ iff $\downarrow S=\downarrow T$. For all $S \in \bar{A}$, $[S]$ denotes its equivalence class. Let $B$ be the set of all equivalence classes. We define an order $\sqsubset$ on $B$ by $[S] \sqsubset[T]$ if and only if $\downarrow S \subsetneq \downarrow T$.

We claim that $\mathcal{B}=\left(B, \sqsubset, \perp_{\sqsubset}\right)$ is a semi-linear order that is closed under finite infima and that the map $\varphi$ given by $\varphi(a) \mapsto[\{a\}]$ is an injective homomorphism of $\mathcal{A}$ in $\mathcal{B}$.

- $\mathcal{B}$ is obviously a partial order. Moreover, note that $\downarrow S$ is a linear and downwards closed suborder of $\mathcal{A}$ for every nonempty finite set $S \subseteq A$. In order to show that $\mathcal{B}$ is semi-linear, assume that $S_{1} \sqsubset S$ and $S_{2} \sqsubset S$. Thus, all elements from $\downarrow S_{1}$ and all elements from $\downarrow S_{2}$ are comparable. Since both sets are downwards closed, this directly implies that either $\left[S_{1}\right]=\left[S_{2}\right],\left[S_{1}\right] \sqsubset\left[S_{2}\right]$ or $\left[S_{2}\right] \sqsubset\left[S_{1}\right]$.

- Let us show that $\mathcal{B}$ is closed under finite infima: Let $S, S_{1}, \ldots, S_{n}$ be finite subsets of $A$ and assume that $[S] \sqsubseteq\left[S_{i}\right]$ for all $1 \leq i \leq n$. Thus, $\downarrow S \subseteq \downarrow S_{i}$. Hence, $\downarrow S \subseteq \bigcap_{i=1}^{n} \downarrow S_{i}=\downarrow \bigcap_{i=1}^{n} S_{i}$. Since $\downarrow \bigcap_{i=1}^{n} S_{i} \subseteq \downarrow S_{i}$ for all $1 \leq \bar{i} \leq n$, $\left[\cap_{i=1}^{n} S_{i}\right]=\inf \left(\left\{\left[S_{1}\right], \ldots\left[S_{n}\right]\right\}\right)$.

- For $a, b \in A$ with $a \neq b$ we have $b \notin \downarrow\{a\}$ or $a \notin \downarrow\{b\}$. Thus, $\varphi$ is an injective map from $A$ to $B$. Moreover, $a<b$ implies $\downarrow\{a\} \subsetneq \downarrow\{b\}$, i.e., $\varphi(a) \sqsubset \varphi(b)$. Similarly, $a \perp b$ implies $a \notin \downarrow\{b\}$ and $b \notin \downarrow\{a\}$, i.e., $\varphi(a) \perp_{\sqsubset} \varphi(b)$.

Lemma 34. Let $\mathcal{A}=(A,<, \perp)$ be a countable semi-linear order that is closed under finite infima. There is a repetition-free enumeration of $\mathcal{A}$, which is closed under infima.

Proof. Fix an arbitrary repetition-free enumeration $\left(a_{i}\right)_{i \in \mathbb{N}}$ of $A$. Assume that we have constructed a sequence $b_{1}, b_{2}, \ldots, b_{i}$ such that $B_{j}=\left\{b_{1}, b_{2}, \ldots, b_{j}\right\}$ is closed under infima for every $j \leq i$. Let $k \in \mathbb{N}$ be minimal with $a_{k} \notin B_{i}$. Let $b_{1}^{\prime}<b_{2}^{\prime}<\ldots b_{m}^{\prime}<a_{k}$ be the list of all infima of the form $\inf \left(S \cup\left\{a_{k}\right\}\right)$ for $S \subseteq B_{i}$ that are not contained in $B_{i}$. This list is indeed linearly ordered by $<$ since all elements in the list are bounded by $a_{k}$. Now set $b_{i+l}=b_{l}^{\prime}$ for all $1 \leq l \leq m$ and set $b_{i+m+1}=a_{k}$. The resulting sequence $b_{1}, \ldots, b_{i+m+1}$ contains $a_{k}$ and $B_{j}=\left\{b_{1}, b_{2}, \ldots, b_{j}\right\}$ is closed under infima for every $j \leq i+m+1$. This can be easily shown using the fact that $\inf (X \cup\{\inf (Y)\})=\inf (X \cup Y)$ for all sets $X$ and $Y$.

Repeating this construction leads to an enumeration $\left(b_{i}\right)_{i \in \mathbb{N}}$ of $A$ with the desired property.

Lemma 35. Let $\mathcal{A}=\left(A, \sqsubset, \perp_{\sqsubset}\right)$ be a countable semi-linear order. There exists an injective homomorphism from $\mathcal{A}$ into $\mathcal{U}$. 
Proof. Due to Lemma 33 and 34 we may assume that $\mathcal{A}$ is closed under finite infima and that $\left(a_{i}\right)_{i \in \mathbb{N}}$ is a repetition-free enumeration of $A$ which is closed under finite infima. Set $A_{i}=\left\{a_{1}, \ldots, a_{i}\right\}$ and $\varphi_{1}: A_{1} \rightarrow U$ with $\varphi_{1}\left(a_{1}\right)=00 \in \mathbb{N} \mathbb{Q}$.

Inductively, we construct injective homomorphisms $\varphi_{i}: A_{i} \rightarrow U$ such that

1. $\varphi_{i+1}$ extends $\varphi_{i}$, and

2. for all $u=n_{1} p_{1} n_{2} p_{2} \ldots n_{k} p_{k} \in \operatorname{im}\left(\varphi_{i}\right)$ and all $1 \leq j \leq k$ we have $p_{j} \in \frac{1}{2^{i}} \mathbb{Z}$.

Assume that $\varphi_{i}$ has already been constructed. We distinguish two cases.

1. If there is some $a \in A_{i}$ with $a_{i+1} \sqsubset a$ let $u=\inf \left\{a \in A_{i} \mid a_{i+1} \sqsubset a\right\}$. Note that $a_{i+1} \sqsubseteq u$. Since the enumeration is closed under infima, we have $u \in A_{i}$ (and thus $\left.a_{i+1} \sqsubset u\right)$ and we can define $\varphi_{i+1}\left(a_{i+1}\right)=\varphi_{i}(u)+\left(\frac{-1}{2^{i+1}}\right)$, where we add according to (5). Note that $\varphi_{i+1}\left(a_{i+1}\right)<\varphi_{i}(u)=\varphi_{i+1}(u)$. In order to prove that this defines a homomorphism, we distinguish the following cases: (a) If $a_{i+1} \sqsubset a$ for some $a \in A_{i}$ then $u \sqsubseteq a$. Hence, $\varphi_{i+1}\left(a_{i+1}\right)<\varphi_{i+1}(u)=$ $\varphi_{i}(u) \leq \varphi_{i}(a)=\varphi_{i+1}(a)$ as desired.

(b) If $a_{i+1} \sqsupset a$ for some $a \in A_{i}$, then $a \sqsubset u$. Hence, $\varphi_{i+1}(a)=\varphi_{i}(a)<$ $\varphi_{i}(u)$. Since $\varphi_{i}$ uses only rationals from $\frac{1}{2^{i}} \mathbb{Z}$, we conclude that $\varphi_{i+1}(a) \leq$ $\varphi_{i}(u)+\frac{-1}{2^{i}}<\varphi_{i+1}\left(a_{i+1}\right)$ as desired.

(c) If $a_{i+1} \perp_{\sqsubset} a$ for some $a \in A_{i}$, then $a \perp_{\sqsubset} u$. By induction, $\varphi_{i+1}(a)=$ $\varphi_{i}(a) \perp_{<} \varphi_{i}(u)=\varphi_{i+1}(u)$. Thus, the assumption $\varphi_{i+1}(a) \leq \varphi_{i+1}\left(a_{i+1}\right)$ leads by transitivity of $\leq$ to the contradiction $\varphi_{i+1}(a) \leq \varphi_{i+1}(u)$. Similarly, the assumption $\varphi_{i+1}(a)>\varphi_{i+1}\left(a_{i+1}\right)$ yields $\varphi_{i+1}(a) \geq \varphi_{i+1}\left(a_{i+1}\right)+$ $\frac{1}{2^{i+1}}=\varphi_{i+1}(u)$. We conclude that $\varphi_{i+1}(a) \perp_{<} \varphi_{i+1}\left(a_{i+1}\right)$ as desired.

2. Otherwise, for all $j \leq i$ we know that $\inf \left\{a_{j}, a_{i+1}\right\}$ is strictly below $a_{i+1}$ and hence belongs to $A_{i}$ (since the enumeration is closed under infima). In particular, the set $\left\{a \in A_{i} \mid a<a_{i+1}\right\}$ is not empty. By semi-linearity, $u=\max \left\{a \in A_{i} \mid a<a_{i+1}\right\}$ is well-defined. Since $\operatorname{im}\left(\varphi_{i}\right)$ is finite, there is some $n \in \mathbb{N}$ such that $\varphi_{i}(u) n 0$ is incomparable to all elements from the set $\varphi_{i}\left(A_{i} \backslash\left\{a \in A_{i} \mid a \leq u\right\}\right)$. Extending $\varphi_{i}$ by setting $\varphi_{i+1}\left(a_{i+1}\right)=\varphi_{i}(u) n 0$ is easily shown to be a homomorphism.

Finally, the limit of $\left(\varphi_{i}\right)_{i \in \mathbb{N}}$ clearly defines an injective homomorphism from $\mathcal{A}$ into $\mathcal{U}$. 